\title{
A selective $p 38 \alpha / \beta$ MAPKs inhibitor alleviates neuropathology and cognitive impairment, and modulates microglia function in 5XFAD mouse
}

\section{Min Sung Gee}

Kyung Hee University

\section{Seung Hwan Son}

Kyung Hee University

\section{Seung Ho Jeon}

Kyung Hee University

Jimin Do

Kyung Hee University

Namkwon Kim

Kyung Hee University

Yeon-Joo Ju

Kyung Hee University

Soo Jin Lee

Kyung Hee University

Eun Kyoung Chung

Kyung Hee University

Kyung-Soo Inn

Kyung Hee University

Nam-Jung Kim

Kyung Hee University

Jong Kil Lee ( $\square$ jklee3984@khu.ac.kr)

Kyung Hee University https://orcid.org/0000-0002-2428-3530

\section{Research}

Keywords: Alzheimer's disease, Amyloid- $\beta$, P38 mitogen-activated protein kinase, Kinase inhibitor, Microglia

Posted Date: March 27th, 2020

DOI: https://doi.org/10.21203/rs.2.21305/v3 
License: (c) (i) This work is licensed under a Creative Commons Attribution 4.0 International License. Read Full License

Version of Record: A version of this preprint was published at Alzheimer's Research and Therapy on April 21 st, 2020. See the published version at https://doi.org/10.1186/s13195-020-00617-2. 


\section{Abstract}

Background: Chronic neuroinflammation, aggressive amyloid beta $(A \beta)$ deposition, neuronal cell loss, and cognitive impairment are pathological presentations of Alzheimer's disease (AD). Therefore, resolution of neuroinflammation and inhibition of $A \beta$-driven pathology have been suggested to be important strategies for $A D$ therapy. Previous efforts to prevent AD progression have identified p38 mitogen-activated protein kinases (MAPKs) as a promising target for AD therapy. Recent studies showed pharmacological inhibition of p38a MAPK improved memory impairment in AD mouse models. Methods: In this study, we used an AD mouse model, 5XFAD, to explore the therapeutic potential of NJK14047 which is a novel,

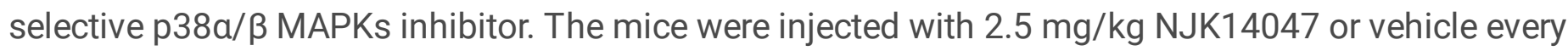
other day for 3 months. Morris water maze task and histological imaging analysis were performed. Protein and mRNA expression levels were measured using immunoblotting and qRT-PCR, respectively. In vitro studies were conducted to measure the cytotoxicity of microglia- and astrocyte-conditioned medium on primary neurons using the MTT assay and TUNEL assay. Results: NJK14047 treatment downregulated phospho-p38 MAPK levels, decreased the amount of A $\beta$ deposits, and reduced spatial learning memory loss in 9-month-old 5XFAD mice. While the pro-inflammatory conditions were decreased, the expression of alternatively activated microglial markers and microglial phagocytic receptors was increased. Furthermore, NJK14047 treatment reduced the number of degenerating neurons labeled with Fluoro-jade B in the brains of 5XFAD mice. The neuroprotective effect of NJK14047 was further confirmed by in vitro studies. Conclusion: Taken together, a selective $p 38 \alpha / \beta$ MAPKs inhibitor NJK14047 successfully showed therapeutic effects for AD in 5XFAD mice. Based on our data, p38 MAPKs inhibition is a potential strategy for AD therapy, suggesting NJK14047 as one of the promising candidates for AD therapeutics targeting p38 MAPKs. Keywords : Alzheimer's disease, Amyloid- $\beta$, P38 mitogen-activated protein kinase, Kinase inhibitor, Microglia

\section{Background}

Alzheimer's disease (AD) is a common progressive neurodegenerative disorder characterized by severe neuronal loss leading to cognitive dysfunction. At present, no effective drug or treatment has been available for the prevention or cure of this disease [1-3]. The major neuropathological feature of $A D$ is the aggregation and deposition of amyloid $\beta(A \beta)$ peptides which are considered one of the major risk factors and causes of $A D[3,4]$. Aggregated $A \beta$ peptides play critical roles in neuronal degeneration, neuroinflammation, and oxidative stress $[5,6]$. Thus, therapeutic approaches for the effective removal of $A \beta$ deposits may provide neuroprotective benefits in $A D$.

According to recently published studies, $A D$ may be exacerbated by neuroinflammation as well as $A \beta$ peptides. Neuroinflammation is mainly mediated by microglial cells which are resident immune cells in the central nervous system (CNS) [6, 7]. Under physiologic conditions, microglial cells are involved in various functions, including the regulation of brain development, the maintenance of homeostasis, and the clearance of old synapses or other debris such as $A \beta$ peptides. In AD, microglia has been known to be chronically activated, resulting in impairment of $A \beta$ clearance, overexpression of pro-inflammatory 
signals, and consequently, neurotoxicity $[8,9]$. Therefore, the regulation of microglial activation may be an important therapeutic strategy of $A D$ by lowering excessive pro-inflammatory immune chemotaxis and enhancing neuroprotective function, leading to the modulation of neuroinflammation $[10,11]$.

Microglial activation plays an important role in several well-known pro-inflammatory signal cascades [12], including those mediated by mitogen-activated protein kinase (MAPK). The MAPK family is a family of serine/threonine protein kinases regulating cell properties in response to extracellular stimuli such as growth factors and inflammatory cytokines [13]. P38 MAPKs, one of the three MAPKs in mammalian cells, are primarily activated by inflammatory cytokines and environmental stresses [14]. P38 MAPKs have four isoforms $a, \beta, \gamma$, and $\delta$ which can be divided into two subgroups; one is $p 38 a$ and $p 38 \beta$, and the other is $p 38 y$ and $p 38 \delta$ [15]. Among these, p38a and p38 $\beta$ MAPKs are highly expressed in adult mouse brain [16]. SB203580, a traditional p38a/ $\beta$ MAPKs inhibitor, had shown therapeutic effects in LPSinduced depression model and AD mouse model $[17,18]$. Recently, two selective p38a MAPK inhibitors, neflamapimod (VX-745) and MW150, also showed therapeutic effects in aged rats and AD mouse model respectively $[19,20]$. The phase 2 a clinical trials of neflamapimod showed improvements in episodic memory in early $A D$ patients $[21,22]$. These results suggest that $p 38 \alpha / \beta$ MAPKs are potentially important targets in $A D$ therapeutics and thus, their inhibitors may be promising drugs.

In our previous study, a novel, selective p38a/ $\beta$ MAPKs inhibitor, NJK14047, could successfully ameliorate microglia-mediated neuroinflammation [23]. It reduced inflammatory responses mediated by lipopolysaccharide (LPS) in the BV2 cells and the mouse model. Based on this finding, we intended to investigate the potential of NJK14047 as a therapeutic agent for AD. In this study, we used five familial Alzheimer's disease (5XFAD) transgenic mice which have been commonly used as AD mouse models. 5XFAD mice overexpress human amyloid precursor protein (hAPP) with three FAD mutations [Swedish (K670N, M671L); Florida (1716V); and London (V717I)] and human presenilin 1 (PS1) with two FAD mutations (M146L and L286V) under the control of the neuron-specific Thy1 promoter [24]. Here, the administration of NJK14047 to the 5XFAD mouse model was suggested to ameliorate memory loss, A $\beta$ deposition, neuroinflammation, and neuronal degeneration via the selective inhibition of $p 38 a / \beta$ MAPKs.

\section{Methods}

Chemicals and reagents. A selective p38a/ $\beta$ MAPKs inhibitor NJK14047 was synthesized by a previously reported procedure (> 97\%, HPLC) $[23,25]$. Dulbecco's modified Eagle's medium (DMEM), fetal bovine serum (FBS), and penicillin/streptomycin were purchased from GE healthcare HyClone ${ }^{\mathrm{TM}}$. Cell culture flasks and plates were purchased from SPL $(70075,30006,30024,30048,30096)$. LPS from Escherichia coli serotype 055:B5 (L6529, $\geq 500,000 \mathrm{EU} / \mathrm{mg}$ ), methylthiazolyldiphenyl-tetrazolium bromide (MTT), thioflavin S (T1892), and DPX mounting medium (06522) were obtained from Sigma-Aldrich. Neurobasal medium (21103-049), B27 supplements (17504-044), RIPA buffer (89901), and protease/phosphatase inhibitor cocktail (78445) were obtained from ThermoFisher Scientific. Fluorescence-mounting medium (S3023) was purchased from Dako. TUNEL (G3250) assay kits were obtained from Promega. Information about antibodies used in this study is listed in Table.1. 
Animals and treatment. 5XFAD mice on B6/SJL background (34840-JAX, Tg6799) and wild type B6/SJL mice from Jackson Laboratory were bred and maintained in an individual ventilated cage with $12 \mathrm{~h}$ light/dark cycles at $22^{\circ} \mathrm{C}$. 5XFAD mice were divided into two experimental groups (treatment vs. vehicle group) using the block randomization method. Similar to previous studies [26, 27], treatment group was treated with NJK14047 at $2.5 \mathrm{mg} / \mathrm{kg}$ every other day from the age of six months to nine months by intraperitoneal injection. Vehicle group and its littermate wild-type mice were treated with the same volume of vehicle by intraperitoneal injection. NJK14047 was dissolved in pure DMSO at $25 \mathrm{mg} / \mathrm{ml}$ which was used as a $40 \mathrm{X}$ stock solution. For working solution, an aliquot of the $40 \mathrm{X}$ stock solution was diluted in PBS and filtered using $0.25 \mu \mathrm{m}$ syringe filter. The body weights of the mice did not significantly differ among groups and any conspicuous side effects (weight loss, anorexia, convulsion, or death) were not observed. All mice used in this study were sacrificed at nine months of age. Both sexes of mice were used in Morris water maze test and $A \beta_{1-42}$ ELSIA without any effect of sex on therapeutic effects. To minimize the sex difference on the degree of amyloidopathy, the protein and RNA samples were obtained from male mice, and histological sections from female mice. All experiments were approved by the Kyung Hee University Institutional Animal Care and Use Committee (IACUC, KHUASP(SE)-17-126-1).

Behavioral test. The Morris water maze task was used to assess spatial memory performance. The water maze was a white circular PVC tank (130-cm diameter, 40-cm height) in which water was filled to $31.5 \mathrm{~cm}$ in depth $\left(23 \pm 1^{\circ} \mathrm{C}\right)$. The submerged target platform was a $10-\mathrm{cm}$ diameter circular zone located $1.5 \mathrm{~cm}$ below the surface of the water. Titanium dioxide $\left(\mathrm{TiO}_{2}\right)$ was dispersed in water to camouflage the target platform. The position of the platform was varied for each mouse with equal distribution between experimental groups. One day before training, mice were habituated to the maze and swimming in the absence of cues. During the training period, mice were subjected to four trials per day, and the start position of each trial was equally distributed. In each trial, the mouse was given $60 \mathrm{~s}$ to find the target platform in the presence of cues around the maze. The time spent finding the platform was recorded as the latency of each trial. If a mouse did not find the platform within $60 \mathrm{~s}$, it was guided to the platform and allowed to stay on the platform for $10 \mathrm{~s}$. After a total 10 days of training, a probe test was performed (day 11). The mouse was subjected to a trial in the absence of the platform for $60 \mathrm{~s}$. The start position was standardized to the opposite direction of the area where the platform was located. All trials were recorded using a camera and analyzed by a free tracking software tool, Toxtrac [28]. The software, the user manual, and the documentation are available at https://toxtrac.sourceforge.io.

Immunoblotting. Immunoblotting analysis was performed as previously described [29]. In brief, mice were sacrificed after the behavioral test. The cortex and hippocampus were quickly isolated and stored at $-80^{\circ} \mathrm{C}$ until use. The brain tissues were homogenized in $10 \mathrm{X}$ volume of lysis buffer (RIPA buffer containing 1\% 100X protease inhibitor) and centrifuged at 13,000 rpm for $20 \mathrm{~min}$. The supernatant was collected and the concentrations were measured using the Bradford method (Bio-Rad, 5000006). Equal amounts of protein samples $(\sim 50 \mu \mathrm{g})$ were fractionated by SDS-PAGE and then, transferred to PVDF membranes. The membranes were blocked with $5 \%$ skim milk and probed with primary antibodies at $4{ }^{\circ} \mathrm{C}$ overnight. After several washes in TBS-T, the membranes were probed with corresponding secondary 
antibodies conjugated with HRP. The immunoblot signals were developed with an enhanced chemiluminescence detection system (ECL, Bio-Rad, 1705061). The band intensity was quantified using Image $\mathrm{J}$ densitometry (NIH, Bethesda, MD, USA), normalized with respect to the $\beta$-actin level.

Tissue preparation and immunofluorescence. The mice were anesthetized with intraperitoneal injection of 2.5\% Avertin (2,2,2-tribromoethanol) and immediately perfused through the heart with PBS followed by $4 \%$ paraformaldehyde in PBS. Brains were excised, post-fixed in $4 \%$ paraformaldehyde at $4^{\circ} \mathrm{C}$ overnight, and incubated in $30 \%$ sucrose at $4^{\circ} \mathrm{C}$ until reaching equilibrium. The brains were embedded in O.C.T. compound blocks at $-80^{\circ} \mathrm{C}$. Sequential $30-\mu \mathrm{m}$ coronal sections were obtained with a cryostat (CM30 50S; Leica). Every tenth section (300 $\mu \mathrm{m}$ apart) of the brain (Bregma -1.30 to $-2.70 \mathrm{~mm}$ ) was used for immunohistochemistry. Free-floating brain sections were rinsed in PBS; blocked for $1 \mathrm{~h}$ in $2 \%$ normal goat serum, $2 \%$ BSA, and $0.4 \%$ Triton-X100; and then incubated with primary antibodies at $4^{\circ} \mathrm{C}$ overnight. After incubation with primary antibodies, the brain sections were washed with PBS and incubated for $2 \mathrm{~h}$ with corresponding secondary antibodies conjugated with Alexa-Fluorescence.

Thioflavin $S$ staining. To stain fibrillary $A \beta$, the brain sections were incubated with $0.1 \%$ thioflavin $S$ in $50 \% \mathrm{EtOH}$ for $10 \mathrm{~min}$. Brain sections were sequentially washed with $50 \% \mathrm{EtOH}, 70 \% \mathrm{EtOH}$, and $100 \% \mathrm{EtOH}$ for 5 min, respectively; afterward, coverslip was applied over fluorescence mounting medium.

$\boldsymbol{A} \boldsymbol{\beta}$ ELISA. An enzyme-linked immunosorbent assay (ELISA) for human $A \beta_{1-42}$ was performed using fluorescence-based ELISA kits (Invitrogen) and appropriate A $\beta$ standards in compliance with the manufacturer's protocol. The hippocampus and frontal cortex from one hemisphere were homogenized in $10 \mathrm{X}$ volume of guanidine buffer with a final concentration of $50 \mathrm{mM}$ Tris and $5 \mathrm{M}$ guanidine $\mathrm{HCl}$ at $\mathrm{pH}$ 8.0. Homogenates were mixed at room temperature for $4 \mathrm{~h}$ and then, diluted in PBS containing $5 \% \mathrm{BSA}$, $0.03 \%$ Tween 20 , and protease inhibitor cocktail.

Quantitative real-time polymerase chain reaction ( $q R T-P C R$ ). Total RNA was extracted from the mouse cortex and hippocampus with a Hybrid-R total RNA purification kit (GeneAll $\left.{ }^{\circledR}, 305-101\right)$ in accordance with the manufacturer's instructions. The NanoDrop ${ }^{T M}$-2000c (ThermoFisher Scientific) was used to assess the concentration and purity of the RNA samples. cDNA was synthesized using TOPscript RT DryMIX (Enzynomics, RT200), according to the manufacturer's instructions. cDNA samples were subjected to qRTPCR using SYBR Green Mix (Enzynomics, RT500) and a CFX Connect real-time PCR system (Bio-Rad).

The qRT-PCR protocol was as follows: first holding stage at $95^{\circ} \mathrm{C}$ for $3 \mathrm{~min}$; followed by a cycling stage at $95^{\circ} \mathrm{C}$ for $10 \mathrm{~s}, 55^{\circ} \mathrm{C}$ for $10 \mathrm{~s}, 72^{\circ} \mathrm{C}$ for $30 \mathrm{~s}$ (30 cycles total); and finally, a holding stage at $95^{\circ} \mathrm{C}$ for $10 \mathrm{~s}$. The information about primers used in this study is listed in Table.2. The measured data were normalized to the GAPDH level using $2^{-\triangle \Delta C T}$ method and expressed as the fold change with respect to the mean of the control group.

Fluoro-jade B staining. To detect late-stage degenerating neuronal cells, Fluoro-jade B staining was performed $[30,31]$. Brain sections were incubated with $1 \% \mathrm{NaOH}$ in $80 \% \mathrm{EtOH}$ for $5 \mathrm{~min}$, followed by sequential washes with $70 \% \mathrm{EtOH}$ and distilled water. Afterward, the brain sections were incubated with 
0.06\% potassium permanganate for $10 \mathrm{~min}$, followed by a 2-min wash with distilled water. The sections were then incubated in $0.0004 \%$ Fluoro-jade B (Millipore, AG310) plus $0.01 \%$ acetic acid solution. After three washes with distilled water, the sections were fully dried and coverslipped with DPX mounting medium.

Cell lines and treatment. The BV2 (RRID: CVCL_0182) murine microglia cell line and the C8-D1A (RRID: CVCL_6379) murine astrocyte cell line were cultured in DMEM with 10\% FBS, $100 \mathrm{U} / \mathrm{ml}$ penicillin, and 100 $\mu \mathrm{g} / \mathrm{ml}$ streptomycin at $37^{\circ} \mathrm{C}$ in a humidified atmosphere of $5 \% \mathrm{CO}_{2}$. Cells were seeded on 6-well plates at $5 \times 10^{5}$ cells/well and stimulated with $500 \mathrm{ng} / \mathrm{ml}$ LPS after $2 \mathrm{~h}$ of pre-treatment with either $1 \mu \mathrm{M}$ or $10 \mu \mathrm{M}$ NJK14047. After $22 \mathrm{~h}$ of LPS stimulation, all media were changed to fresh neurobasal medium. The cells were incubated in neurobasal media for another $24 \mathrm{~h}$. The conditioned neurobasal medium was obtained and centrifuged at 1,500 rpm for 5 min to remove the remaining cells. The supernatant was collected and stored at $4^{\circ} \mathrm{C}$, and then used to culture primary neurons within $24 \mathrm{~h}$.

Primary cells and treatment. Primary mouse cortical microglia and astrocytes were prepared according to the commonly used protocols as previously described [32,33]. Briefly, mouse cortical mixed glia was obtained from P1 to P4 C57BL/6J mouse pups and seeded on poly-L-lysine pre-coated 75T flasks at 1.5 $\times 10^{7}$ cells/flask. Culture medium was changed every three days. After five to seven days, the flasks were vigorously tapped, and the supernatant was collected and centrifuged at 3,000 rpm for 10 min to obtain microglia. The cell pellet was dispersed in new medium and seeded on poly-L-lysine pre-coated 6-well plates at $5 \times 10^{5}$ cells/well. After $2 \mathrm{~h}$ of cell seeding, the culture medium was replaced with fresh medium to remove the oligodendrocytes. Approximately $96 \%$ of the cells were found to be positive for ionized calcium-binding adapter molecule 1 (lba-1) which is a marker of microglia. Afterward, the flasks were tapped, and the supernatant was discarded; the rest of the cells in the flask were rinsed with PBS and seeded onto poly-L-lysine pre-coated 6-well plates at $5 \times 10^{5}$ cells/well to harvest astrocytes.

Approximately $98 \%$ of the cells were positive for glial fibrillary acidic protein (GFAP) which is a marker of astrocytes. Prepared microglia and astrocytes were stimulated with $50 \mathrm{ng} / \mathrm{ml}$ LPS after $2 \mathrm{~h}$ of pretreatment with $10 \mu \mathrm{M}$ NJK14047. After $22 \mathrm{~h}$ of LPS stimulation, all media were changed to fresh neurobasal medium to incubate the cells for another $24 \mathrm{~h}$. The conditioned neurobasal medium was obtained and centrifuged at 1,500 rpm for $5 \mathrm{~min}$. The supernatant was collected stored at $4^{\circ} \mathrm{C}$, and then treated to primary neurons within $24 \mathrm{~h}$.

Primary mouse cortical neurons were prepared as previously described [34]. In brief, mouse cortical neurons were obtained from E17 C56BL/6J mouse embryos. The neurons were seeded on poly-L-lysine pre-coated 48-well plates or 24-well coverslips for the MTT and the TUNEL assay, respectively.

Neurobasal medium containing 2\% B27 supplement, 2 mM L-glutamine (Welgene, LS 002-01), and 1\% $\mathrm{P} / \mathrm{S}$ was used to culture neurons. Primary neurons were used in experiments at 10 days in vitro (DIV).

Cytotoxicity assay. The MTT assay is generally used to assess the cell viability by measuring the metabolic activity of the cells [35]. To assess the cytotoxicity of glia-conditioned medium, mouse cortical primary neurons were seeded on poly-L-lysine pre-coated 48 -well plates at $1 \times 10^{5}$ cells/well. On DIV10, 
neurons were incubated in the medium conditioned with BV2, C8-D1A, primary cultured microglia, and primary cultured astrocytes, respectively, for $24 \mathrm{~h}$. The media were replaced with neurobasal media containing 10\% MTT. Absorbance at $570 \mathrm{~nm}$ corrected with $690 \mathrm{~nm}$ values were measured by a microplate reader. The measured cell viability was expressed as a percentage relative to the control mean.

TUNEL assay. Apoptotic DNA fragmentation was detected using the TUNEL method [36]. To measure the apoptotic cell death of primary neuronal cells, mouse cortical primary neurons were seeded on poly-Llysine pre-coated 24-well cover glasses at $1.5 \times 10^{5}$ cells/well. On DIV10, neurons were incubated in each conditioned medium for $24 \mathrm{~h}$. After incubation, the cells were subjected to TUNEL staining in accordance with the manufacturer's instructions. Apoptotic cells were detected as localized bright green cells (positive cells) in a blue background using an Olympus BX51 microscope. The extent of apoptosis was quantified using Image $\mathrm{J}$ software.

Confocal microscopy and image analysis. All stained brain sections were imaged by confocal microscopy. Z-stacked images were acquired at 1.5- $\mu \mathrm{m}$ intervals (total 15 optical slices). Four cortex areas area (2 left; 2 right) and four hippocampus areas area (2 left; 2 right) were imaged in one brain slice. For each mouse, thioflavin S-positive area, 6E10-positive area, and Fluoro-jade B-positive cells were quantified with four to five brain slices (Bregma -1.30 to $-2.70,300 \mu \mathrm{m}$ apart) using Image $\mathrm{J}$ software. The number of Iba-1- and GFAP-positive cells were quantified using Cell Profiler software [37]. To quantify the number of plaques associated microglia, Iba-1-positive cells within the $20 \mu \mathrm{m}$ range of plaques were manually counted. A total of 70-75 plaques $(>10 \mu \mathrm{m})$ from five mice per group were measured [26].

Statistical analysis. In all in vivo studies, $\mathrm{n}$ represents the number of animals used in the corresponding experiment. For in vitro studies, $\mathrm{n}$ is the number of independent experiments. The group size for each experiment was based on our previous results [38]. The operators responsible for the experimental procedure and data analysis were blinded and unaware of group allocation throughout the experiments. All data were analyzed using SPSS version 25 (IBM corporation, NY, USA) with statistical significance defined as a $P$-value less than 0.05 . Parametric tests such as ANOVA were used when the data satisfied the null hypothesis of the Levene's test. The Tukey's post-hoc test was performed if the P-value was < 0.05 in one-way ANOVA. In case of qRT-PCR and the MTT assay, the Kruskal-Wallis test followed by Dunn's post-hoc multiple comparisons was used to compare among the experimental groups. Latency of Morris water maze task was analyzed using the generalized estimating equation (GEE) method. Descriptive statistics were used to summarize the data using mean \pm standard error of the mean (SEM). All graphs were constructed using Graph Pad Prism 5.0 software (Graph Pad software Inc., CA, USA).

\section{Results}

\section{NJK14047 as a selective p38a/ $\beta$ MAPKs inhibitor reduces the level of phospho-p38 MAPKs in the brain and attenuates spatial memory loss in 9-month-old 5XFAD mice.}


The phospho-p38 MAPKs level was upregulated in the brain of 9-month-old 5XFAD mice in both cortex and hippocampus regions of the brain compared to the wild-type mice (Fig. 1A). The upregulated phospho-p38 MAPKs levels were significantly reduced in the NJK14047-treated 5XFAD mouse brain to the level comparable to that of the WT mice. This inhibitory effect was observed in both cortex and hippocampus regions (Fig 1A).

Based on the suppressive effects of NJK14047 on the p38 MAPKs pathway in 5XFAD mice, we further evaluated whether NJK14047 treatment could ameliorate spatial learning memory impairment in 5XFAD mice. NJK14047- and vehicle-treated 5XFAD mice as well as their wild-type littermates were subjected to Morris water maze task [39]. As shown in Figure 1B, overall, 5XFAD mice showed markedly longer escape latency than wild-type mice $(P<0.05$ since day 6$)$. However, the escape latency was significantly reduced with NJK14047 treatment compared to the control 5XFAD mice $(P<0.05$ since day 8) (Fig. 1B). At day 11, a probe task was performed with the hidden platform removed. Representative swimming paths of each group during the probe task were shown in Figure 1B. 5XFAD mice showed less total swimming distance compared to the wild-type littermates without significant difference between NJK14047- and vehicletreated groups (Fig. 1C). The recorded time spent in the target quadrant was $24.48 \pm 2.14 \mathrm{~s}$ in wild-type mice and $13.14 \pm 2.35 \mathrm{~s}$ in 5XFAD mice $(P<0.01)$. The elapsed time in the target quadrant was significantly increased in the NJK14047-treated 5XFAD mice $(23.79 \pm 2.08 \mathrm{~s}, P<0.01$ vs. 5XFAD mice without NJK14047 treatment) (Fig. 1D), which was comparable to the wild-type mice. Target zone cross also differed between groups in a 60-s probe task. Wild-type group and 5XFAD + NJK14047 group showed $4.25 \pm 0.77$ times and $4.00 \pm 0.62$ times, respectively, whereas the frequency of crossing the target zone was significantly lower in the 5XFAD group without treatment $(1.50 \pm 0.57$ times, $P<0.05)$ (Fig. 1E). These results suggest NJK14047 treatment reduce phohpo-p38 MAPK levels in the 5XFAD mouse brains and ameliorates spatial learning memory loss in 9-month-old 5XFAD mice.

\section{NJK14047 decreases A $\beta$ deposits in the brain of 9-month-old 5XFAD mice.}

As a next step, we investigated whether $A \beta$ deposits, one of the representative hallmarks in $A D$, were altered with NJK14047 treatment in the 5XFAD mice. As shown in Figure 2A, thioflavin S-positive A $\beta$ plaques were observed in 9-month-old 5XFAD mice; while no plaque was detected in their wild-type littermates. Notably, the thioflavin S-positive area was decreased in both cortex and hippocampus regions of NJK14047 treated 5XFAD mice. Immunostaining with a 6 E10 antibody against human $A \beta_{1-16}$ showed similar results, supporting the reduction of $A \beta$ plaques by NJK14047 treatment in the brain (Fig. 2B). This $A \beta$ reduction as a result of NJK14047 administration was further confirmed using human $A \beta_{1-42}$ ELISA kit (Fig. 2C). According to these data, NJK14047 treatment attenuates A $\beta$ deposits in the 9-month-old 5XFAD mouse brain.

\section{A $\beta$ processing and degradation are not affected by NJK14047.}

Reduction of $A \beta$ plaques in the NJK14047-treated mouse brain might result from the decrease in the APP production or the reduction in the level of proteins related to $A \beta$ processing or degradation. To investigate 
this hypothesis, the levels of hAPP, BACE1, and PS1 proteins were examined by immunoblotting. No significant change in hAPP, BACE1, and PS1 levels was observed in NJK14047-treated mouse brains compared to those in 5XFAD mouse brains, suggesting the decrease in A $\beta$ plaques by NJK14047 does not result from the reduction in APP processing (Fig. 3A). In addition to APP processing molecules, proteins involved in $A \beta$ degradation, such as insulin degrading enzyme (IDE) and neutral endopeptidase (neprilysin, NEP), could also regulate A $\beta$ deposits. $[40,41]$. Similar to APP processing molecules, the protein levels of $A \beta$ degrading enzymes were not significantly different among the experimental groups (Fig. 3B). These results suggest NJK14047 treatment could not directly alter the APP processing pathway or $A \beta$ degrading enzymes in 9-month-old 5XFAD mice.

\section{NJK14047 inhibits neuroinflammatory conditions in the brains of 9-month-old 5XFAD mice.}

Because neuroinflammation has been suggested to contribute to $A D$ pathogenesis and is related to $A \beta$ production and elimination [6, 42], we examined the neuroinflammation state in our 5XFAD mice. First, we assessed the gliosis of microglia and astrocytes in 5XFAD mice. As expected, 9-month-old 5XFAD mice exhibited higher extent of microgliosis than wild-type littermates in the cortex and hippocampus. This upregulated microgliosis was significantly decreased in NJK14047-treated 5XFAD mice (Fig 4A). Similar to microgliosis, increased astrogliosis was decreased in the NJK14047-treated 5XFAD group (Fig 4B). For further investigation, the microglial activation markers were analyzed using qRT-PCR. Consistent with the gliosis results, the expression levels of pro-inflammatory cytokines such as tumor necrosis factor a (TNFa), interleukin $1 \beta$ (IL-1 $\beta$ ), and interleukin 6 (IL-6) were significantly elevated in the 5XFAD mouse brains compared to those in the wild-type brains (Fig. 4C, upper). On the other hand, the levels of arginase 1 (Arg1), chil3 chitinase-like 3 (Chi3l3/YM-1), and resistin like alpha (Retnla/Fizz-1), which indicate alternative active state of microglia, were not significantly different between the wild-type and 5XFAD mice except Fizz-1 (Fig. 4C, lower). The expression levels of pro-inflammatory cytokines were significantly decreased in the NJK14047-treated 5XFAD mouse brains except TNF-a (Fig. 4C, upper). On the contrary, Arg1, YM-1, and Fizz-1 were upregulated in the brain cortex of NJK14047-treated 5XFAD mice. In the hippocampus, Arg1 and YM-1 expression levels were apparently increased in the 5XFAD + NJK14047 group without reaching statistical significance; for Fizz-1, the expression level was significantly increased compared to the 5XFAD group (Fig. 4C, lower). Taken together, these results indicate NJK14047 treatment alleviates inflammatory conditions in the 9-month-old 5XFAD mouse brain.

\section{NJK14047 increases phagocytic activity of microglia in association with Aß clearance.}

The alternative activation of microglia was previously shown to be effective for phagocytosis and degradation of $A \beta$ peptides [43]. To further confirm the alleviation of the neuroinflammatory condition in 5XFAD mice as a result of NJK14047 treatment, we investigated the phagocytic function of microglia in 9-month-old 5XFAD mice. The mRNA expression levels of the following phagocytic receptors in the brain were measured using qRT-PCR: macrophage receptor with collagenous structure (MACRO); macrophage scavenger receptor 1 (Msr1); scavenger receptor class B, member 1 (Scarb1); and scavenger receptor class B, member 3 (Scarb3). As expected, NJK14047-treated 5XFAD mice showed increased expression 
levels of phagocytic receptors compared to wild-type or vehicle-treated 5XFAD mice except Scarb1 (Fig. $5 A)$. For histological examination, the brain sections were co-stained with thioflavin S and Iba-1 antibody. Consistent with qRT-PCR data, microglia were primarily found to cluster around A $\beta$ plaques in the NJK14047-treated mouse brains (Fig. 5B). These data indicate NJK14047 treatment induces antiinflammatory responses and improves microglial phagocytosis of $A \beta$ peptides in 9-month-old 5XFAD mice.

\section{NJK14047 reduces the neuronal cell death in the cortex and hippocampus.}

According to previous studies, $A \beta$ deposits and chronic neuroinflammation may provoke neuronal cell death, contributing to important pathology in AD [44-47]. In this present study, A $\beta$ plaques and neuroinflammation were substantially decreased in NJK14047-treated 9-month-old 5XFAD mice; therefore, we tried to confirm our hypothesis that the p38 MAPK inhibitor might reduce the neuronal cell death in 5XFAD mice. As expected, we observed Fluoro-jade B-positive cells in the cortex and hippocampus of 9-month-old 5XFAD mice, but not in wild-type mice. Pretreatment with NJK14047 in the 5XFAD mice led to fewer Fluoro-jade B-positive cells in both brain regions compared to those without pretreatment (Fig. 6). This finding is consistent with our speculation that neuronal cell death could be decreased in association with the reduction in $A \beta$ plaques as well as alleviation of neuroinflammatory state in the 5XFAD mouse brains, which might result from the pharmacological inhibition of $p 38 a / \beta$ MAPKs.

\section{NJK14047 decreases neurotoxicity mediated by the activated microglia.}

To delineate the relations between neuroinflammation suppression by NJK14047 and the neuroprotective effect in AD mice, the potential pharmacological effects of NJK14047 were evaluated using primary neurons, microglial cell line BV2, and astrocyte cell line C8-D1A. BV2 and C8-D1A were cultured in vitro, and the conditioned media (CM) of BV2 and C8-D1A were prepared as described in "Materials and methods." The mouse primary cortical neurons were treated with each CM for $24 \mathrm{~h}$ to assess the cell viability (Fig 7A). As shown in Fig 7B, we observed neurotoxicity of LPS-stimulated BV2 CM. However, pretreatment of NJK14047 in BV2 cells alleviated the neurotoxicity induced by LPS-stimulated BV2 CM (Fig. 7B, left). In contrast, LPS-stimulated C8-D1A CM showed no neurotoxicity (Fig. 7B, right). These cell viability results were confirmed in TUNEL assay; TUNEL-positive neurons were increased in the group treated with LPS-stimulated BV2 CM, but decreased in the NJK14047-treated BV2 CM group (Fig. 7C).

The decreased neurotoxicity of LPS-stimulated BV2 CM with NJK14047 treatment was further confirmed using primary microglia and astrocytes. Primary microglia conditioned medium (MCM) and astrocyte conditioned medium (ACM) were prepared as described in "Materials and methods." Primary neurons were incubated with each CM for $24 \mathrm{~h}$ and subjected to the MTT and TUNEL assay (Fig. 8A). Similar to our cell line study, LPS-stimulated MCM produced neurotoxicity which was reversed by NJK14047 treatment; however, no changes were observed with ACM (Fig. 8B). Consistent with the MTT assay, more TUNEL-positive cells were detected in the LPS-stimulated MCM group in comparison to the naïve MCM group; NJK14047 treatment resulted in much fewer TUNEL-positive cells than the untreated group. As 
expected, ACM-treated neurons showed no significant alterations (Fig 8C). Overall, based on these findings, reactive microglia, rather than astrocytes, provoke the apoptosis of neurons, and NJK14047 can attenuate neurotoxicity of LPS-stimulated MCM.

\section{Discussion And Conclusions}

5XFAD mouse is a transgenic AD mouse model which is characterized by rapid accumulation of $A \beta$ in the brain, leading to chronic neuroinflammation. This model exhibits $A \beta$ aggregation and gliosis from 2 months of age, followed by neuronal loss from 6 months of age [24]. 5XFAD mice showed spatial learning memory impairment after 9 months of age [48] and motor function deficits after 12 months of age [49]. In the present study, we showed NJK14047 treatment from 6-month-old 5XFAD mice could significantly ameliorate $A \beta$-induced neuroinflammation, thereby alleviating neuronal degeneration and cognitive impairment in the 9-month-old 5XFAD mice.

P38 MAPKs inhibition is one of the promising targets for AD therapy. Numerous studies have attempted to attenuate $A \beta$-induced neurotoxicity and memory impairment through inhibition of the p38 MAPKs signaling [50,51]. Recent reports using selective p38a MAPK inhibitors, neflamapimod (VX-745), MW150, and MW181, have shown potential therapeutic effects for AD. Neflamapimod (VX-745) improved water maze performance in aged rats [19] and enhanced episodic memory in early AD patients [21, 22]. In addition, MW150 was reported to show therapeutic effects in APP/PS1 mice at the age of 11 to 12 months [20,52]. Furthermore, MW181 was suggested to reduce tau-related pathologic features and improve working memory in 20-month-old hTau mice [53]. In this study, we presented the therapeutic effects of a selective p38a/ $\beta$ MAPKs inhibitor NJK14047 and investigated its mode of action in 9-monthold 5XFAD mice. P38 MAPKs has been well known to be activated in chronic inflammatory conditions such as $A D$. Consistently, several studies have shown the up-regulation of phospho-p38 levels in the brains of AD mouse models, but not in 5XFAD mice. We demonstrated upregulation of the phospho-p38 levels in the cortex and hippocampus of 9-month-old 5XFAD mice, which could be reversed by NJK14047. Although blood-brain barrier (BBB) is a major obstacle for many drugs targeting the brain, NJK14047 successfully achieved its inhibitory effect on p38 MAPKs in the cortex and hippocampus of 5XFAD mice. In fact, it is plausible that the effect of NJK14047 might be attributed to the damaged BBB of old 5XFAD mice due to chronic inflammatory conditions. The low molecular weight $(M W=445.515)$ and lipophilic property (cLogP $=3.12$ ) of NJK14047 might also contribute to sufficient penetration into the brain. Further experiments are warranted to determine whether NJK14047 can cross the intact BBB.

Down-regulation of the phospho-p38 MAPKs level is related to the decreased neuroinflammation, which can reverse reactive gliosis and overexpression of pro-inflammatory cytokines such as TNF- $\alpha$, IL-1 $\beta$, and IL-6. According to a recent study using the p38a MAPK inhibitor MW150 in the APP/PS1 mice at the age of 11 to 12 months, IL-1 $\beta$ and TNF-a levels were decreased; microglial cell counts were increased around the plaque [20]. Similarly, we confirmed NJK14047, reduced the pro-inflammatory cytokines and upregulated alternatively activated microglial markers such as Arg1, YM-1, and Fizz-1 in the cortex and hippocampus of 5XFAD mice. In addition, NJK14047 treatment increased the mRNA expression of 
microglial phagocytic receptors in the brain and improved the number of plaque associated microglia, suggesting the recovery of microglial phagocytic activity [54]. In neuroinflammatory conditions, resident microglia or infiltrated macrophages were able to polarize into the pro-inflammatory phenotype (M1) upon exposure to pro-inflammatory cytokines or protein debris. These cells express high levels of proinflammatory cytokines and reactive oxygen species, which can be toxic to neurons. In contrast, the alternative activation of microglia (M2) has been previously reported to have positive influences on tissue repair, immunological regulation, and phagocytosis-mediated $A \beta$ clearance [43, 55]. Thus, NJK14047 treatment could alter the ratio of $M 1$ to $M 2$ microglia population, ultimately ameliorating $A D$ pathology. However, this dichotomous classification of in vivo microglia into M1 and M2 may be oversimplified [56]. More specifically elaborated analysis and classification are required to evaluate the microglial variation with NJK14047 treatment.

The process of generating A $\beta$ peptide from APP could be mediated by the $\beta$-secretase and $\gamma$-secretase complex; in $A D$, the produced $A \beta$ is accumulated in the extracellular matrix. $A \beta$ peptides could be degraded by proteolytic enzymes such as IDE, NEP, and MMP-9 or phagocytosed by microglia or astrocytes. Our study demonstrated NJK14047 treatment reduced $A \beta$ accumulation in 9-month-old 5XFAD mice. However, no significant differences in APP processing enzymes or A $\beta$ degradation enzymes were observed with NJK14047 treatment. According to recent studies regarding p38a MAPK in AD mouse models, genetic modulation of neuronal p38a MAPK could ameliorate pathologic features associated with $A D[57,58]$. They showed down-regulation of p38a MAPK in neurons reduced BACE1 activity, thereby lowering $A \beta$ accumulation in the mice with early stage $A D$. Interestingly, Colié et al. reported no significant alterations in the BACE1 protein levels between the experimental groups in 12-month-old 5XFAD mice, possibly indicating variable effects of neuronal p38a inhibition on the BACE1 levels depending on the age of 5XFAD mice [57]. Based on these results, NJK14047 might inhibit the neuronal p38a/ $\beta$ MAPKs and decrease the BACE1 levels in the early phase of treatment; however, these effects might be diminished in 9-month-old mice used in this study. Additionally, our current study showed increased phagocytic receptors of microglia and the number of microglia around $A \beta$ plaque, ameliorating neuroinflammatory conditions. The enhanced microglial phagocytic function might also contribute to the decrease in $A \beta$ accumulation.

Another possible cause of $A \beta$ reduction and restoration of microglial phagocytic function is inhibition of p38 $\beta$ MAPK by NJK14047. According to a previous study, both p38a and p38 $\beta$ MAPKs were highly active in the basal brain condition. However, $\mathrm{p} 38 \mathrm{a}$ and $\mathrm{p} 38 \beta$ showed different localization, indicating their potentially distinct roles or mechanisms. In particular, p38 $\beta$ was detected in glial cells as well as neuronal nuclei in many brain regions [16]. Thus, $p 38 \beta$ MAPK inhibition might be important to successfully ameliorate the inflammatory conditions of glial cells, suggesting dual inhibition of both $p 38 a$ and $p 38 \beta$ as an effective therapeutic strategy for AD. Further studies are needed to elaborate this theory.

During the final stage of $A D$, irreversible neuronal loss occurs in the cortex and hippocampus regions which play a role encoding of new information and consolidation of memory networks. In this regard, protection of neurons against harmful factors such as $A \beta$ plaques or massive pro-inflammatory signals is 
important for AD therapy. In this study, spatial learning memory was significantly improved in NJK14047treated 5XFAD mice compared to vehicle-treated 5XFAD mice. Moreover, neuronal degeneration was significantly decreased in the brains of NJK14047-treated mice. Furthermore, the neuroprotective effects of NJK14047 were observed in in vitro studies; NJK14047 treatment reversed the neuronal apoptosis caused by LPS-stimulated BV2 CM. Interestingly, LPS-stimulated C8-D1A CM showed no toxicity to the tested neurons. This neuroprotective effect of NJK14047 was further investigated using primary microglia and astrocytes. Consistent with cell line studies, apoptosis occurred in the neurons cultured in the LPS-stimulated MCM; however, this neurotoxicity was alleviated by pre-treatment with NJK14047. Similar to C8-D1A, LPS-stimulated ACM showed no apparent toxicity to neurons. Although recent studies have reported the toxic effects of reactive astrocytes with $A 1$ phenotype on neurons, polarization into $A 1$ astrocytes requires activated MCM including IL-1a, TNF, and C1q [59]. Collectively, LPS-stimulated astrocytes without pro-inflammatory microglial activation are suggested to be less toxic to neurons, and inhibition of microglial activation by NJK14047 may contribute to neuroprotection.

In summary, we have shown pharmacological inhibition of $p 38 a / \beta$ MAPKs in the brain of 5XFAD mice contributes to reduction of $A \beta$ accumulation as well as amelioration of neuroinflammatory conditions and microglial phagocytic functions; as a result, neuronal death was decreased, and learning memory was improved. As NJK14047 generally acts on neurons and glia by inhibiting both $p 38 a$ and $p 38 \beta$ MAPKs, further studies are warranted to evaluate which p38 MAPK accounts for the therapeutic effects of NJK14047 in neurons and glia. Taken together, a selective p38a/ $\beta$ MAPKs inhibitor NJK14047 successfully showed therapeutic effects in 5XFAD mice. Our data support the potential of p38 MAPKs inhibition as a treatment strategy for $A D$, suggesting NJK14047 might be one of the promising candidates for $A D$ therapeutics targeting p38 MAPKs.

\section{Abbreviations}

$A \beta$, amyloid beta; $A C M$, astrocyte conditioned medium; AD, Alzheimer's disease; Arg1, arginase 1; BACE1, beta-site APP cleaving enzyme 1; BBB, blood-brain barrier; Chi3l3/YM-1, chil3 chitinase-like 3; CM, conditioned medium; GFAP, glial fibrillary acidic protein; hAPP, human amyloid precursor protein; Iba-1, ionized calcium-binding adapter molecule 1; IDE, insulin degrading enzyme; MACRO, macrophage receptor with collagenous structure; MCM, microglia conditioned medium; Msr1, macrophage scavenger receptor 1; NEP, neprilysin; PS1, presenilin 1; Retnla/Fizz-1, resistin like alpha; Scarb1, scavenger receptor class $B$, member 1 ; Scarb3, scavenger receptor class $B$, member 3

\section{Declarations}

\section{Ethics approval and consent to participate}

All animal experiments were conducted in compliance with the Guide for the Care and Use of Laboratory Animals of the National Institutes of Health and were approved by the Kyung Hee University Institutional Animal Care and Use Committee (IACUC, KHUASP(SE)-17-126-1). 


\section{Consent for publication}

Not applicable

\section{Availability of data and materials}

The datasets used and/or analyzed during the current study are available from the corresponding author on reasonable request.

\section{Competing Interests}

The authors declare no competing interest.

\section{Funding}

This research was supported by the Basic Science Research Program through the National Research Foundation of Korea (NRF) funded by the Ministry of Education (NRF-2018R1D1A1B07050547) as well as the Ministry of Science, ICT \& Future Planning (NRF-2017R1A5A2014768).

\section{Author Contributions}

M.S.G and S.H.S performed the experiments, analyzed the data, and prepared the manuscript. S.H.J and Y.J.J performed the animal study. J.D and N.K provided mouse primary cells. S.J.L synthesized and provided NJK14047. K.S.I, N.J.K, and J.K.L interpreted the data and reviewed the manuscript. E.K.C performed the statistical analysis. K.S.I, N.J.K, and J.K.L. designed the study and wrote the manuscript. All authors discussed the results and commented on the manuscript.

\section{Acknowledgements}

Not applicable

\section{References}

1. Huang Y, Mucke L: Alzheimer mechanisms and therapeutic strategies. Cell 2012, 148(6):1204-1222.

2. Gouras GK, Almeida CG, Takahashi RH: Intraneuronal Abeta accumulation and origin of plaques in Alzheimer's disease. Neurobiology of aging 2005, 26(9):1235-1244.

3. Yiannopoulou KG, Papageorgiou SG: Current and future treatments for Alzheimer's disease. Therapeutic advances in neurological disorders 2013, 6(1):19-33.

4. Murphy MP, LeVine H, 3rd: Alzheimer's disease and the amyloid-beta peptide. Journal of Alzheimer's disease : JAD 2010, 19(1):311-323.

5. Yang TT, Hsu CT, Kuo YM: Cell-derived soluble oligomers of human amyloid-beta peptides disturb cellular homeostasis and induce apoptosis in primary hippocampal neurons. Journal of neural transmission (Vienna, Austria : 1996) 2009, 116(12):1561-1569. 
6. Cai Z, Hussain MD, Yan LJ: Microglia, neuroinflammation, and beta-amyloid protein in Alzheimer's disease. The International journal of neuroscience 2014, 124(5):307-321.

7. Kim YS, Joh TH: Microglia, major player in the brain inflammation: their roles in the pathogenesis of Parkinson's disease. Experimental \& molecular medicine 2006, 38(4):333-347.

8. Clayton KA, Van Enoo AA, Ikezu T: Alzheimer's Disease: The Role of Microglia in Brain Homeostasis and Proteopathy. Frontiers in neuroscience 2017, 11:680.

9. Hansen DV, Hanson JE, Sheng M: Microglia in Alzheimer's disease. The Journal of cell biology 2018, 217(2):459-472.

10. Ardura-Fabregat A, Boddeke E, Boza-Serrano A, Brioschi S, Castro-Gomez S, Ceyzeriat K, Dansokho C, Dierkes T, Gelders G, Heneka MT et al: Targeting Neuroinflammation to Treat Alzheimer's Disease. CNS drugs 2017, 31(12):1057-1082.

11. McManus RM, Heneka MT: Role of neuroinflammation in neurodegeneration: new insights. Alzheimer's research \& therapy 2017, 9(1):14.

12. Kaminska B, Gozdz A, Zawadzka M, Ellert-Miklaszewska A, Lipko M: MAPK signal transduction underlying brain inflammation and gliosis as therapeutic target. Anatomical record (Hoboken, NJ : 2007) 2009, 292(12):1902-1913.

13. Pearson G, Robinson F, Beers Gibson T, Xu BE, Karandikar M, Berman K, Cobb MH: Mitogen-activated protein (MAP) kinase pathways: regulation and physiological functions. Endocrine reviews 2001, 22(2):153-183.

14. Johnson GL, Lapadat R: Mitogen-activated protein kinase pathways mediated by ERK, JNK, and p38 protein kinases. Science (New York, NY) 2002, 298(5600):1911-1912.

15. Cuenda A, Rousseau S: p38 MAP-kinases pathway regulation, function and role in human diseases. Biochimica et biophysica acta 2007, 1773(8):1358-1375.

16. Lee SH, Park J, Che Y, Han PL, Lee JK: Constitutive activity and differential localization of p38alpha and p38beta MAPKs in adult mouse brain. Journal of neuroscience research 2000, 60(5):623-631.

17. Zhao YW, Pan YQ, Tang MM, Lin WJ: Blocking p38 Signaling Reduces the Activation of Proinflammatory Cytokines and the Phosphorylation of p38 in the Habenula and Reverses DepressiveLike Behaviors Induced by Neuroinflammation. Frontiers in pharmacology 2018, 9:511.

18. Yao Y, Huang JZ, Chen L, Chen Y, Li X: In vivo and in vitro studies on the roles of p38 mitogenactivated protein kinase and NADPH-cytochrome P450 reductase in Alzheimer's disease. Experimental and therapeutic medicine 2017, 14(5):4755-4760.

19. Alam JJ: Selective Brain-Targeted Antagonism of p38 MAPKalpha Reduces Hippocampal IL-1beta Levels and Improves Morris Water Maze Performance in Aged Rats. Journal of Alzheimer's disease : JAD 2015, 48(1):219-227.

20. Zhou Z, Bachstetter AD, Spani CB, Roy SM, Watterson DM, Van Eldik LJ: Retention of normal glia function by an isoform-selective protein kinase inhibitor drug candidate that modulates cytokine production and cognitive outcomes. Journal of neuroinflammation 2017, 14(1):75. 
21. Alam J, Blackburn K, Patrick D: Neflamapimod: Clinical Phase 2b-Ready Oral Small Molecule Inhibitor of p38alpha to Reverse Synaptic Dysfunction in Early Alzheimer's Disease. The journal of prevention of Alzheimer's disease 2017, 4(4):273-278.

22. Scheltens P, Prins N, Lammertsma A, Yaqub M, Gouw A, Wink AM, Chu HM, van Berckel BNM, Alam J: An exploratory clinical study of p38alpha kinase inhibition in Alzheimer's disease. Annals of clinical and translational neurology 2018, 5(4):464-473.

23. Gee MS, Kim SW, Kim N, Lee SJ, Oh MS, Jin HK, Bae JS, Inn KS, Kim NJ, Lee JK: A Novel and Selective p38 Mitogen-Activated Protein Kinase Inhibitor Attenuates LPS-Induced Neuroinflammation in BV2 Microglia and a Mouse Model. Neurochemical research 2018, 43(12):2362-2371.

24. Oakley H, Cole SL, Logan S, Maus E, Shao P, Craft J, Guillozet-Bongaarts A, Ohno M, Disterhoft J, Van Eldik $L$ et al: Intraneuronal beta-amyloid aggregates, neurodegeneration, and neuron loss in transgenic mice with five familial Alzheimer's disease mutations: potential factors in amyloid plaque formation. The Journal of neuroscience : the official journal of the Society for Neuroscience 2006, 26(40):10129-10140.

25. Heo J, Shin H, Lee J, Kim T, Inn KS, Kim NJ: Synthesis and biological evaluation of Ncyclopropylbenzamide-benzophenone hybrids as novel and selective p38 mitogen activated protein kinase (MAPK) inhibitors. Bioorganic \& medicinal chemistry letters 2015, 25(17):3694-3698.

26. Pan RY, Ma J, Kong XX, Wang XF, Li SS, Qi XL, Yan YH, Cheng J, Liu Q, Jin W et al: Sodium rutin ameliorates Alzheimer's disease-like pathology by enhancing microglial amyloid-beta clearance. Science advances 2019, 5(2):eaau6328.

27. An J, Zhou Y, Zhang M, Xie Y, Ke S, Liu L, Pan X, Chen Z: Exenatide alleviates mitochondrial dysfunction and cognitive impairment in the 5XFAD mouse model of Alzheimer's disease. Behavioural brain research 2019, 370:111932.

28. Rodriguez A, Zhang H, Klaminder J, Brodin T, Andersson M: Toxld: an efficient algorithm to solve occlusions when tracking multiple animals. Sci Rep 2017, 7(1):14774.

29. Lee JK, Jin HK, Endo S, Schuchman EH, Carter JE, Bae J-s: Intracerebral Transplantation of Bone Marrow-Derived Mesenchymal Stem Cells Reduces Amyloid-Beta Deposition and Rescues Memory Deficits in Alzheimer's Disease Mice by Modulation of Immune Responses. Stem cells (Dayton, Ohio) 2010, 28(2):329-343.

30. Schmued LC, Hopkins KJ: Fluoro-Jade B: a high affinity fluorescent marker for the localization of neuronal degeneration. Brain research 2000, 874(2):123-130.

31. Poirier JL, Capek R, De Koninck Y: Differential progression of Dark Neuron and Fluoro-Jade labelling in the rat hippocampus following pilocarpine-induced status epilepticus. Neuroscience 2000, 97(1):59-68.

32. Tamashiro TT, Dalgard CL, Byrnes KR: Primary microglia isolation from mixed glial cell cultures of neonatal rat brain tissue. Journal of visualized experiments : JoVE 2012(66):e3814.

33. Lian H, Roy E, Zheng H: Protocol for Primary Microglial Culture Preparation. Bio-protoco/ 2016, 6(21). 
34. Lee JK, Jin HK, Bae JS: Bone marrow-derived mesenchymal stem cells attenuate amyloid betainduced memory impairment and apoptosis by inhibiting neuronal cell death. Current Alzheimer research 2010, 7(6):540-548.

35. Stockert JC, Horobin RW, Colombo LL, Blazquez-Castro A: Tetrazolium salts and formazan products in Cell Biology: Viability assessment, fluorescence imaging, and labeling perspectives. Acta histochemica 2018, 120(3):159-167.

36. Gorczyca W, Bruno S, Darzynkiewicz R, Gong J, Darzynkiewicz Z: DNA strand breaks occurring during apoptosis - their early insitu detection by the terminal deoxynucleotidyl transferase and nick translation assays and prevention by serine protease inhibitors. International journal of oncology 1992, 1(6):639-648.

37. Carpenter AE, Jones TR, Lamprecht MR, Clarke C, Kang IH, Friman O, Guertin DA, Chang JH, Lindquist RA, Moffat $\mathrm{J}$ et al: CellProfiler: image analysis software for identifying and quantifying cell phenotypes. Genome Biology 2006, 7(10):R100.

38. Kim N, Do J, Ju IG, Jeon SH, Lee JK, Oh MS: Picrorhiza kurroa Prevents Memory Deficits by Inhibiting NLRP3 Inflammasome Activation and BACE1 Expression in 5xFAD Mice. Neurotherapeutics : the journal of the American Society for Experimental NeuroTherapeutics 2020, 17(1):189-199.

39. Vorhees CV, Williams MT: Morris water maze: procedures for assessing spatial and related forms of learning and memory. Nature protocols 2006, 1(2):848-858.

40. Kurochkin IV, Goto S: Alzheimer's beta-amyloid peptide specifically interacts with and is degraded by insulin degrading enzyme. FEBS letters 1994, 345(1):33-37.

41. Iwata N, Tsubuki S, Takaki Y, Watanabe K, Sekiguchi M, Hosoki E, Kawashima-Morishima M, Lee HJ, Hama E, Sekine-Aizawa $Y$ et al: Identification of the major Abeta1-42-degrading catabolic pathway in brain parenchyma: suppression leads to biochemical and pathological deposition. Nature medicine 2000, 6(2):143-150.

42. Heneka MT, Carson MJ, El Khoury J, Landreth GE, Brosseron F, Feinstein DL, Jacobs AH, Wyss-Coray T, Vitorica J, Ransohoff RM et al: Neuroinflammation in Alzheimer's disease. The Lancet Neurology 2015, 14(4):388-405.

43. Cherry JD, Olschowka JA, O'Banion MK: Neuroinflammation and M2 microglia: the good, the bad, and the inflamed. Journal of neuroinflammation 2014, 11:98.

44. Chen WW, Zhang X, Huang WJ: Role of neuroinflammation in neurodegenerative diseases (Review). Molecular medicine reports 2016, 13(4):3391-3396.

45. Skaper SD, Facci L, Zusso M, Giusti P: An Inflammation-Centric View of Neurological Disease: Beyond the Neuron. Frontiers in cellular neuroscience 2018, 12:72.

46. Bayer TA, Wirths O: Intracellular accumulation of amyloid-Beta - a predictor for synaptic dysfunction and neuron loss in Alzheimer's disease. Frontiers in aging neuroscience 2010, 2:8.

47. Morishima Y, Gotoh Y, Zieg J, Barrett T, Takano H, Flavell R, Davis RJ, Shirasaki Y, Greenberg ME: $\boldsymbol{\beta}$ Amyloid Induces Neuronal Apoptosis Via a Mechanism that Involves the c-Jun N-Terminal Kinase Pathway and the Induction of Fas Ligand. The Journal of Neuroscience 2001, 21(19):7551-7560. 
48. Schneider F, Baldauf K, Wetzel W, Reymann KG: Behavioral and EEG changes in male 5xFAD mice. Physiology \& behavior 2014, 135:25-33.

49. O'Leary TP, Robertson A, Chipman PH, Rafuse VF, Brown RE: Motor function deficits in the 12 monthold female 5xFAD mouse model of Alzheimer's disease. Behavioural brain research 2018, 337:256263.

50. Lee JK, Kim NJ: Recent Advances in the Inhibition of p38 MAPK as a Potential Strategy for the Treatment of Alzheimer's Disease. Molecules (Basel, Switzerland) 2017, 22(8).

51. Kheiri G, Dolatshahi M, Rahmani F, Rezaei N: Role of p38/MAPKs in Alzheimer's disease: implications for amyloid beta toxicity targeted therapy. Reviews in the neurosciences 2018, 30(1):9-30.

52. Roy SM, Grum-Tokars VL, Schavocky JP, Saeed F, Staniszewski A, Teich AF, Arancio O, Bachstetter $A D$, Webster SJ, Van Eldik LJ et al: Targeting human central nervous system protein kinases: An isoform selective p38alphaMAPK inhibitor that attenuates disease progression in Alzheimer's disease mouse models. ACS chemical neuroscience 2015, 6(4):666-680.

53. Maphis N, Jiang S, Xu G, Kokiko-Cochran ON, Roy SM, Van Eldik LJ, Watterson DM, Lamb BT, Bhaskar K: Selective suppression of the alpha isoform of p38 MAPK rescues late-stage tau pathology. Alzheimer's research \& therapy 2016, 8(1):54.

54. Wilkinson K, El Khoury J: Microglial scavenger receptors and their roles in the pathogenesis of Alzheimer's disease. International journal of Alzheimer's disease 2012, 2012:489456.

55. Orihuela R, McPherson CA, Harry GJ: Microglial M1/M2 polarization and metabolic states. British journal of pharmacology 2016, 173(4):649-665.

56. Ransohoff RM: A polarizing question: do M1 and M2 microglia exist? Nature neuroscience 2016, 19(8):987-991.

57. Colie S, Sarroca S, Palenzuela R, Garcia I, Matheu A, Corpas R, Dotti CG, Esteban JA, Sanfeliu C, Nebreda AR: Neuronal p38alpha mediates synaptic and cognitive dysfunction in an Alzheimer's mouse model by controlling beta-amyloid production. Scientific reports 2017, 7:45306.

58. Schnoder L, Hao W, Qin Y, Liu S, Tomic I, Liu X, Fassbender K, Liu Y: Deficiency of Neuronal p38alpha MAPK Attenuates Amyloid Pathology in Alzheimer Disease Mouse and Cell Models through Facilitating Lysosomal Degradation of BACE1. The Journal of biological chemistry 2016, 291(5):2067-2079.

59. Liddelow SA, Guttenplan KA, Clarke LE, Bennett FC, Bohlen CJ, Schirmer L, Bennett ML, Munch AE, Chung WS, Peterson TC et al: Neurotoxic reactive astrocytes are induced by activated microglia. Nature 2017, 541(7638):481-487.

\section{Tables}

Table.1 Information of immunostaining antibodies used in this study 


\begin{tabular}{|c|c|c|c|c|c|}
\hline Target & Host & Source & Catalog no. & RRID & Application \\
\hline phospho-p38 MAPK & Rabbit & Cell Signaling Technology & 9215 & AB_331762 & WB / 1:1000 \\
\hline p38 MAPK & Rabbit & Cell Signaling Technology & 9212 & AB_330713 & WB / 1:1000 \\
\hline $\begin{array}{l}\text { Human } \beta \text {-amyloid 1-16 } \\
\text { (clone: 6E10) }\end{array}$ & Mouse & BioLegend & 803001 & AB_2564653 & $\begin{array}{l}\text { WB / 1:1000 } \\
\text { IHC / 1:500 }\end{array}$ \\
\hline BACE1 & Mouse & Millipore & MAB5308 & AB_11212616 & WB / 1:1000 \\
\hline Presenilin-1 & Rabbit & Cell Signaling Technology & 5643 & AB_10706356 & WB / 1:1000 \\
\hline IDE & Rabbit & Abcam & ab32216 & AB_775686 & WB / 1:1000 \\
\hline NEP & Goat & R\&D System & AF1126 & AB_2144426 & WB / 1:1000 \\
\hline Iba-1 & Rabbit & Wako & 019-19741 & AB_839504 & IHC / 1:500 \\
\hline GFAP & Chicken & Abcam & ab4674 & AB_304558 & IHC / 1:500 \\
\hline Lipocalin-2 & Rabbit & Abcam & ab63929 & AB_1140965 & $\begin{array}{l}\text { WB / 1:1000 } \\
\text { IHC / 1:500 }\end{array}$ \\
\hline$\beta$-actin (HRP) & Mouse & Santa Cruz Biotechnology & sc-47778 HRP & AB_2714189 & WB / 1:5000 \\
\hline Mouse IgG (HRP) & Goat & Santa Cruz Biotechnology & sc-2005 & AB_631736 & WB / 1:5000 \\
\hline Rabbit IgG (HRP) & Goat & Santa Cruz Biotechnology & sc-2054 & AB_631748 & WB / 1:5000 \\
\hline Goat IgG (HRP) & Donkey & Santa Cruz Biotechnology & sc-2020 & AB_631728 & WB / 1:5000 \\
\hline Mouse IgG (488) & Goat & Invitrogen & A11001 & AB_2534069 & IHC / 1:1000 \\
\hline Rabbit IgG (488) & Goat & Invitrogen & A11008 & AB_143165 & IHC / 1:1000 \\
\hline Rabbit IgG (594) & Goat & Invitrogen & A11012 & AB_2534079 & IHC / 1:1000 \\
\hline Chicken IgY (488) & Goat & Invitrogen & A11039 & AB_2534096 & IHC / 1:1000 \\
\hline
\end{tabular}

WB, Western blot; IHC, Immunohistochemistry.

Table.2 Information of qRT-PCR primers used in this study

\begin{tabular}{llll}
\hline Gene name & NCBI reference & Forward primer $\left(5^{\prime} \rightarrow 3^{\prime}\right)$ & Reverse primer $\left(5^{\prime} \rightarrow 3^{\prime}\right)$ \\
\hline TNF- $\alpha$ & NM_013693 & GATTATGGCTCAGGGTCCAA & GCTCCAGTGAATTCGGAAAG \\
IL-1 $\beta$ & NM_008361 & CCCAAGCAATACCCAAAGAA & GCTTGTGCTCTGCTTGTGAG \\
\hline IL-6 & NM_031168 & CCGGAGAGGAGACTTCACAG & TTGCCATTGCACAACTCTTT \\
\hline Arg1 & NM_007482 & AGTTGGGTTCACTTCCATGA & CGATTCACCTGAGCTTTGAT \\
\hline YM-1 & NM_009892 & AGAGCAAGAAACAAGCATGG & CTGTACCAGCTGGGAAGAAA \\
\hline Fizz & NM_020509 & TCCAGCTAACTATCCCTCCACTGT & GGCCCATCTGTTCATAGT \\
\hline MACRO & NM_010766 & CTGTGGCAATGGATCACTAGC & CTCCTGGCTGGTATGGACC \\
\hline Msr1 & NM_031195 & TGAACGAGAGGATGCTGACTG & GGAGGGGCCATTTTAGTGC \\
\hline SCARB1 & NM_016741 & TTTGGAGTGGTAGTAAAAAGGGC & TGACATCAGGGACTCAGAGTAG \\
\hline SCARB3 & NM_007643 & GAACCACTGCTTTCAAAAACTGG & TGCTGTTCTTTGCCACGTCA \\
\hline GAPDH & NM_008084 & TGAATACGGCTACAGCAACA & AGGCCCCTCCTGTTATTATG \\
\hline
\end{tabular}

\section{Figures}


A

Cortex

Hippocampus
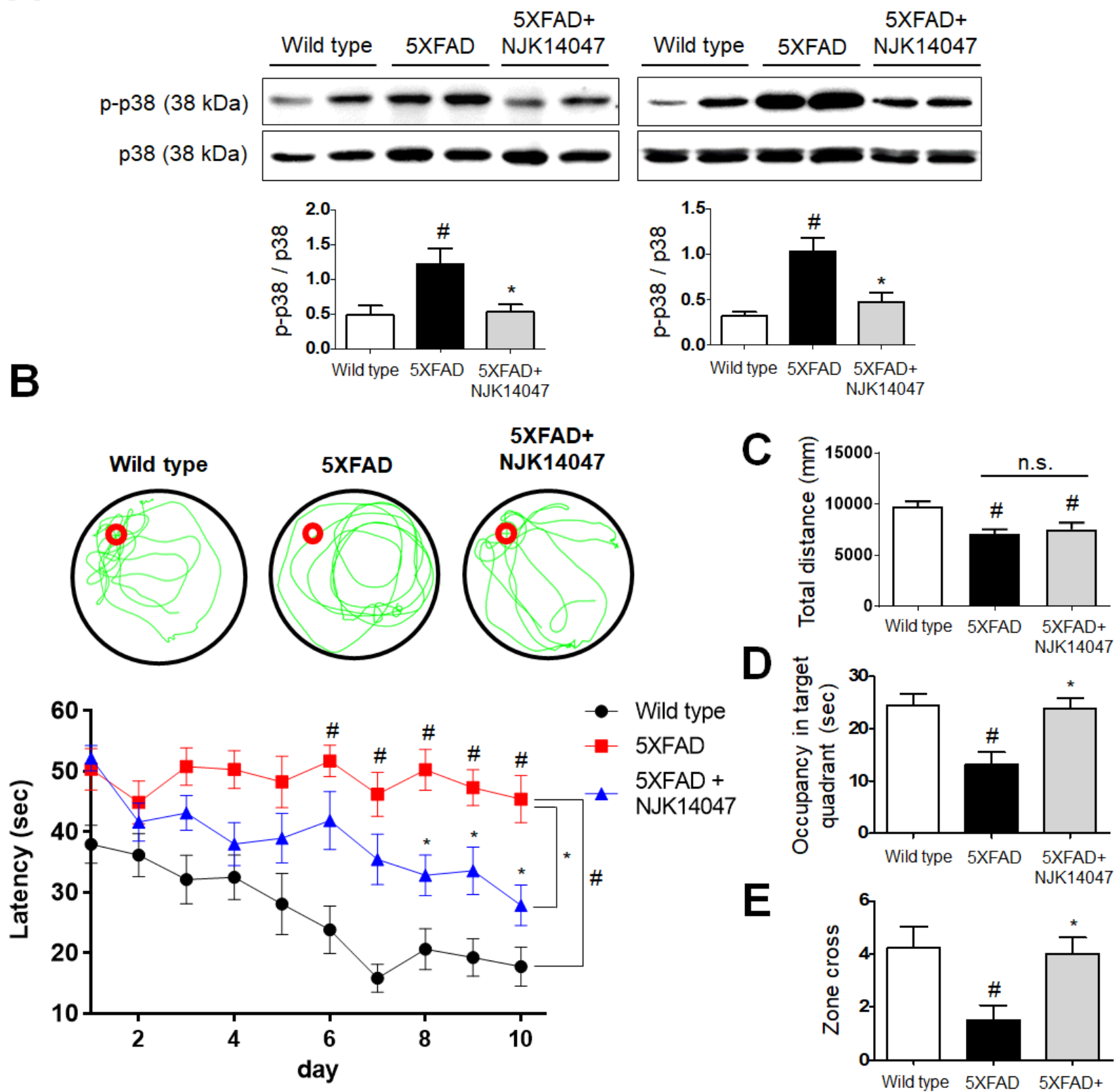

E

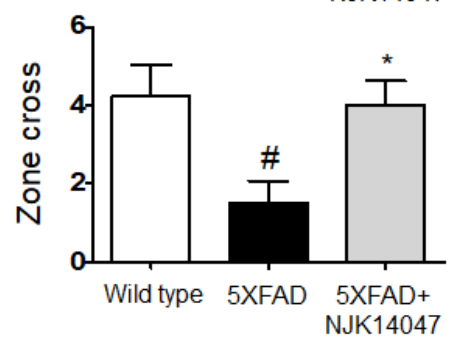

\section{Figure 1}

NJK14047 treatment from 6 months to 9 months of age reduces phospho-p38 MAPK level and prevents spatial learning memory impairment. (A) Inhibition of p38 MAPK in the cortex and hippocampus of NJK14047-treated 9-month-old 5XFAD mice. Phospho-p38 protein level was normalized to total p38 level and shown as mean \pm SEM ( $n=6$ per group, one-way ANOVA). (B) Morris water maze task between three groups; wild-type, 5XFAD and 5XFAD + NJK14047. Representative swimming paths during a probe task 
after 10 days of training and escape latencies of each group for total 10 days of training. Data are shown as mean \pm SEM ( $n=12-13$ per group, GEE analysis). (C-E) After 10 days of training, a probe task was performed for the mice. (C) Total swimming distance was measured without significant difference between the 5XFAD and 5XFAD + NJK14047 group. (D) Time spent in the target quadrant and (E) the frequency of crossing the target zone during a 60-second probe test. Data are shown as mean \pm SEM ( $n=$ 12-13 per group, one-way ANOVA). (\#P $<0.05$ vs. wild type; *P $<0.05$ vs. 5XFAD)

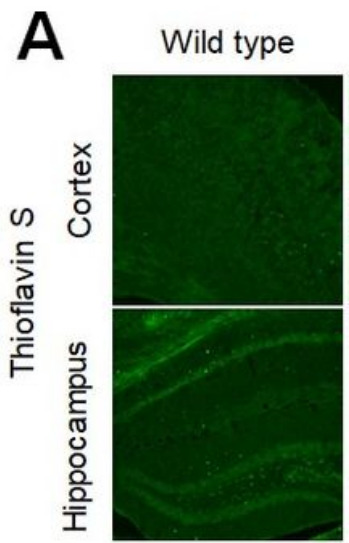

Cortex

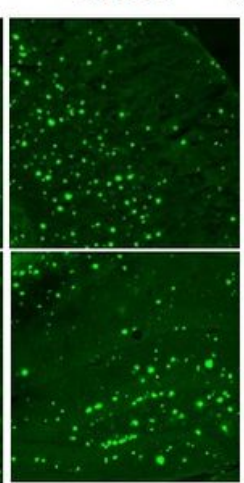

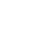
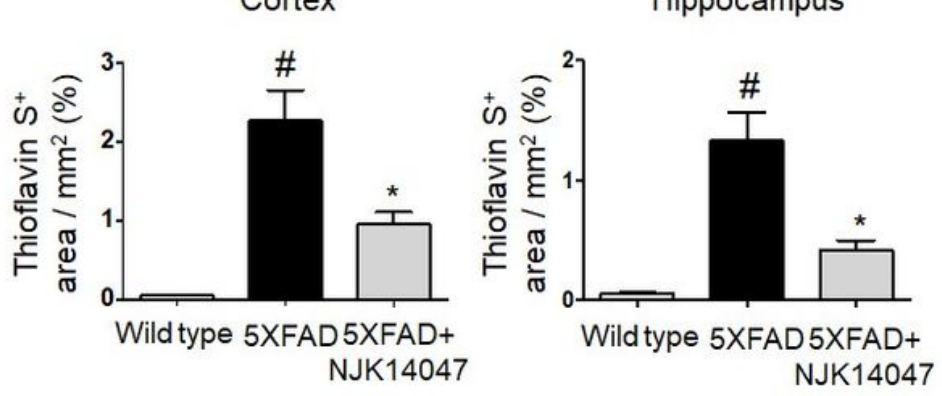

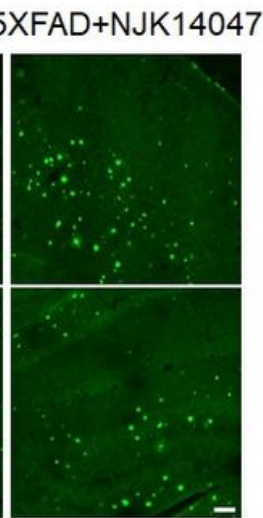

Hippocampus

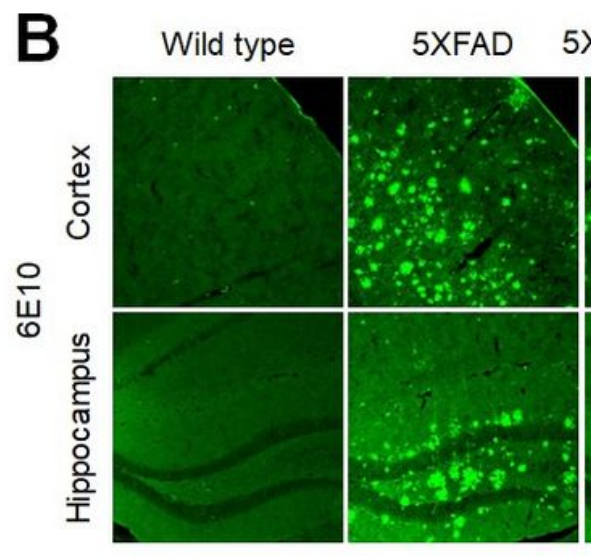

Cortex

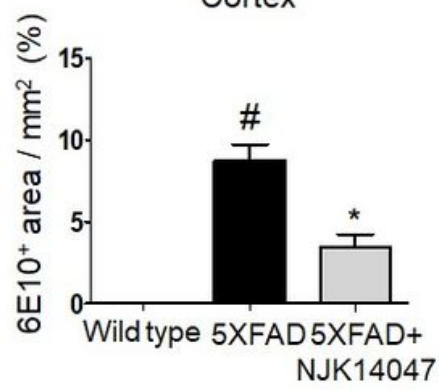

5XFAD+NJK14047

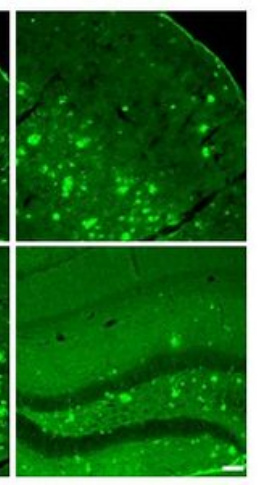

Hippocampus

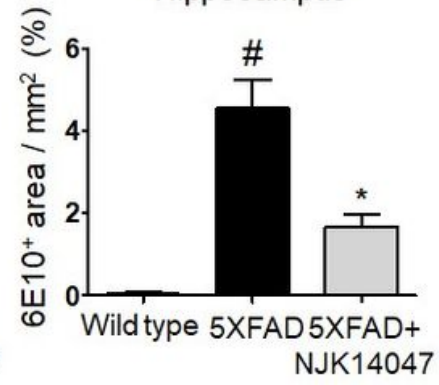

C

Cortex

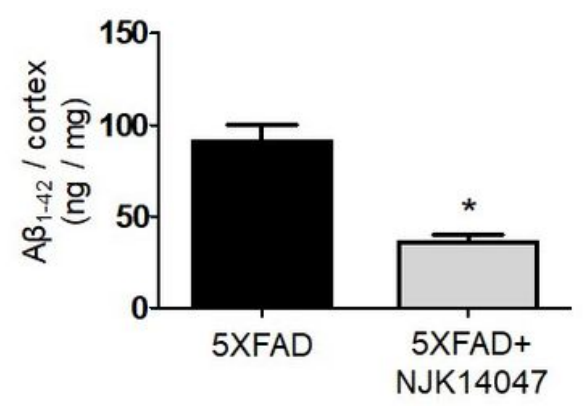

Hippocampus

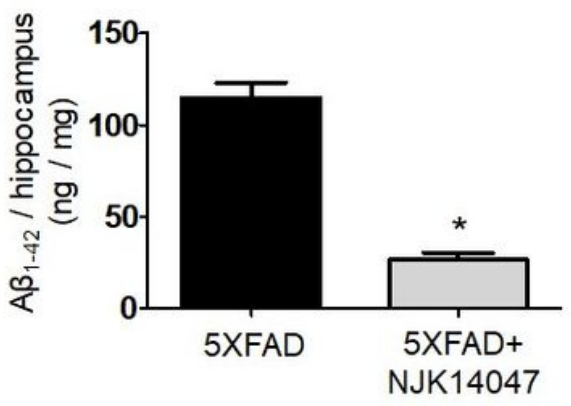

Figure 2

NJK14047 treatment from 6 months to 9 months of age reduces $A \beta$ plaques in the cortex and hippocampus. (A and B) Representative confocal microscope images of mouse brain slices stained with (A) thioflavin S dye and (B) 6E10 antibody. Thioflavin S- and 6E10-positive areas per square millimeter were quantified. For quantification, four areas in each section and four sections in each mouse were 
used. Data are shown as mean \pm SEM (scale bar $=100 \mu \mathrm{m}, \mathrm{n}=5$ per group, one-way ANOVA). (C) A $\beta$ protein level per brain cortex and hippocampus weight $(\mathrm{mg})$ was measured using A $\beta 1-42$ ELISA. Data are shown as mean \pm SEM ( $n=6$ per group, Student's t-test). (\#P< 0.05 vs. wild type; ${ }^{*} P<0.05$ vs. 5XFAD)
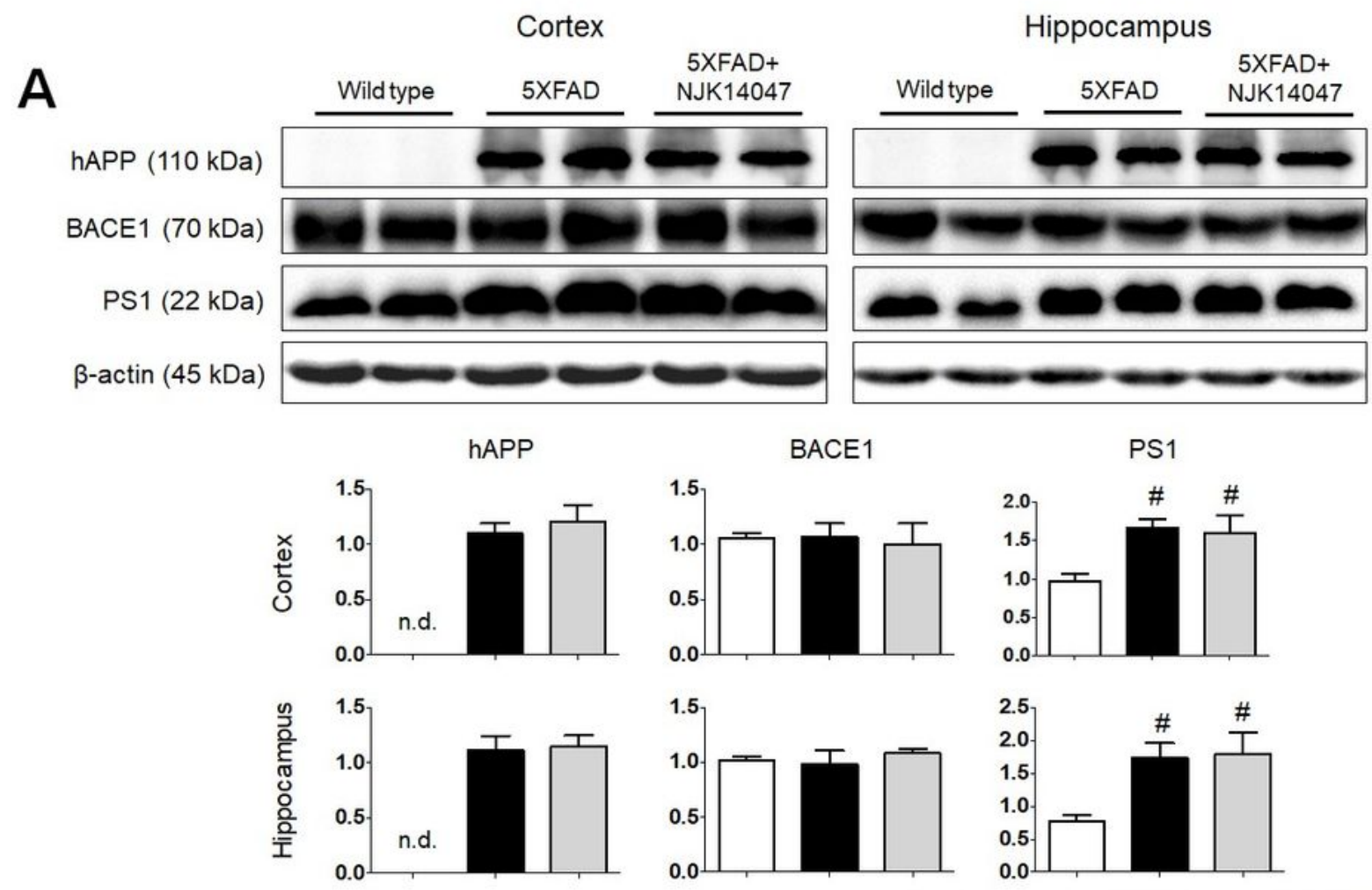

Wild type

5XFAD $\square$ 5XFAD+NJK14047

B

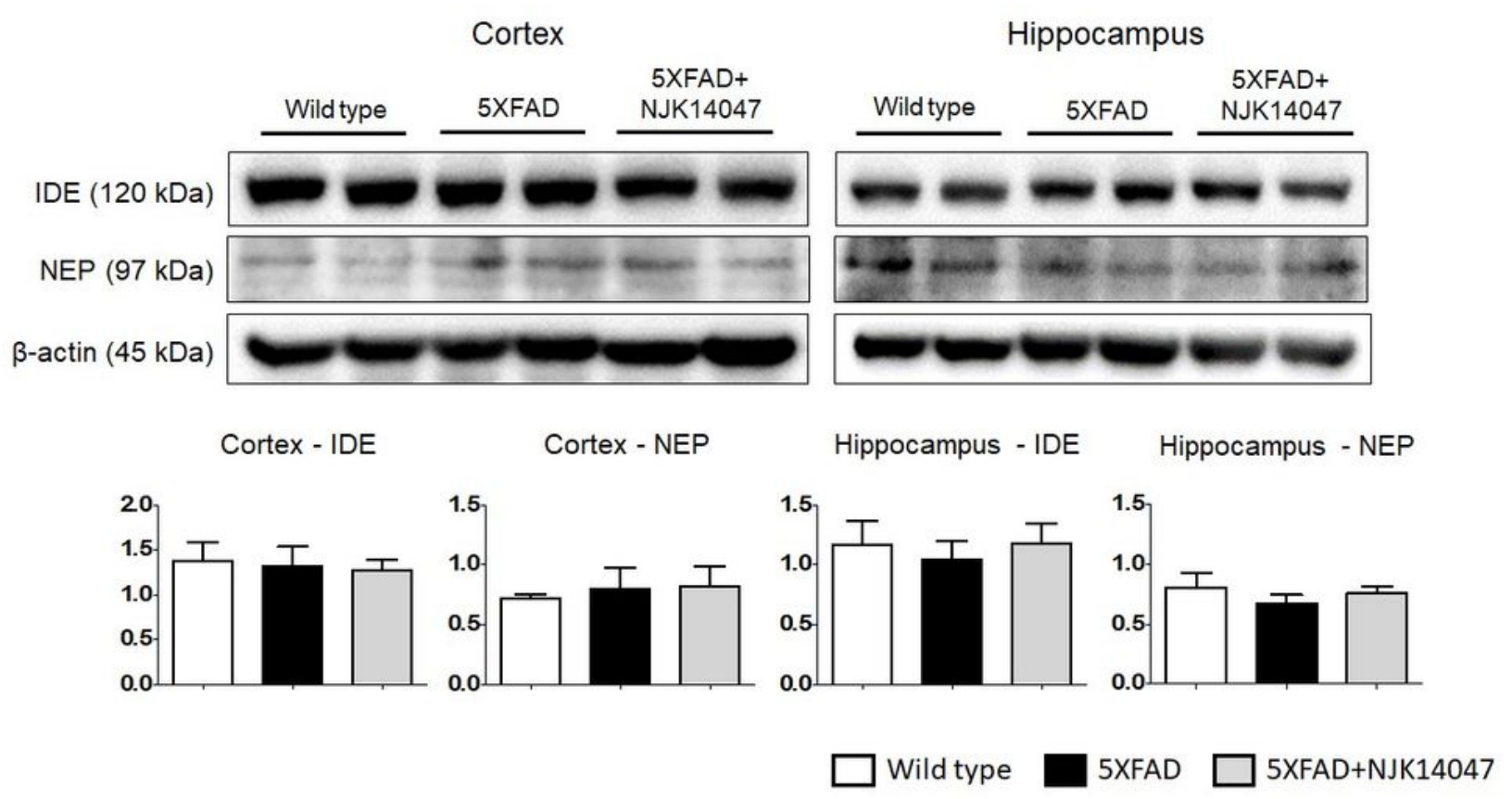

Figure 3

NJK14047 treatment from 6 months to 9 months of age does not affect amyloid beta precursor protein processing and degrading molecules. (A) Representative immunoblotting images and quantifications for 
proteins involved in the production of $A \beta$. Total protein lysates of the cortex and hippocampus were used and immunoblotted with 6E10, BACE1, and PS1 antibodies. Protein levels were quantified using Image J, normalized to its $\beta$-actin level. Data were shown as mean \pm SEM ( $n=5$ per group, one-way ANOVA). (B) Representative immunoblotting images and quantifications for proteins involved in the elimination of $A \beta$. Immunoblotting was performed with IDE and NEP antibodies using total protein lysates of the cortex and hippocampus. Quantitative analysis was performed using Image J. The quantified protein levels were normalized to the $\beta$-actin level and expressed as mean \pm SEM $(n=5$ per group, one-way ANOVA).

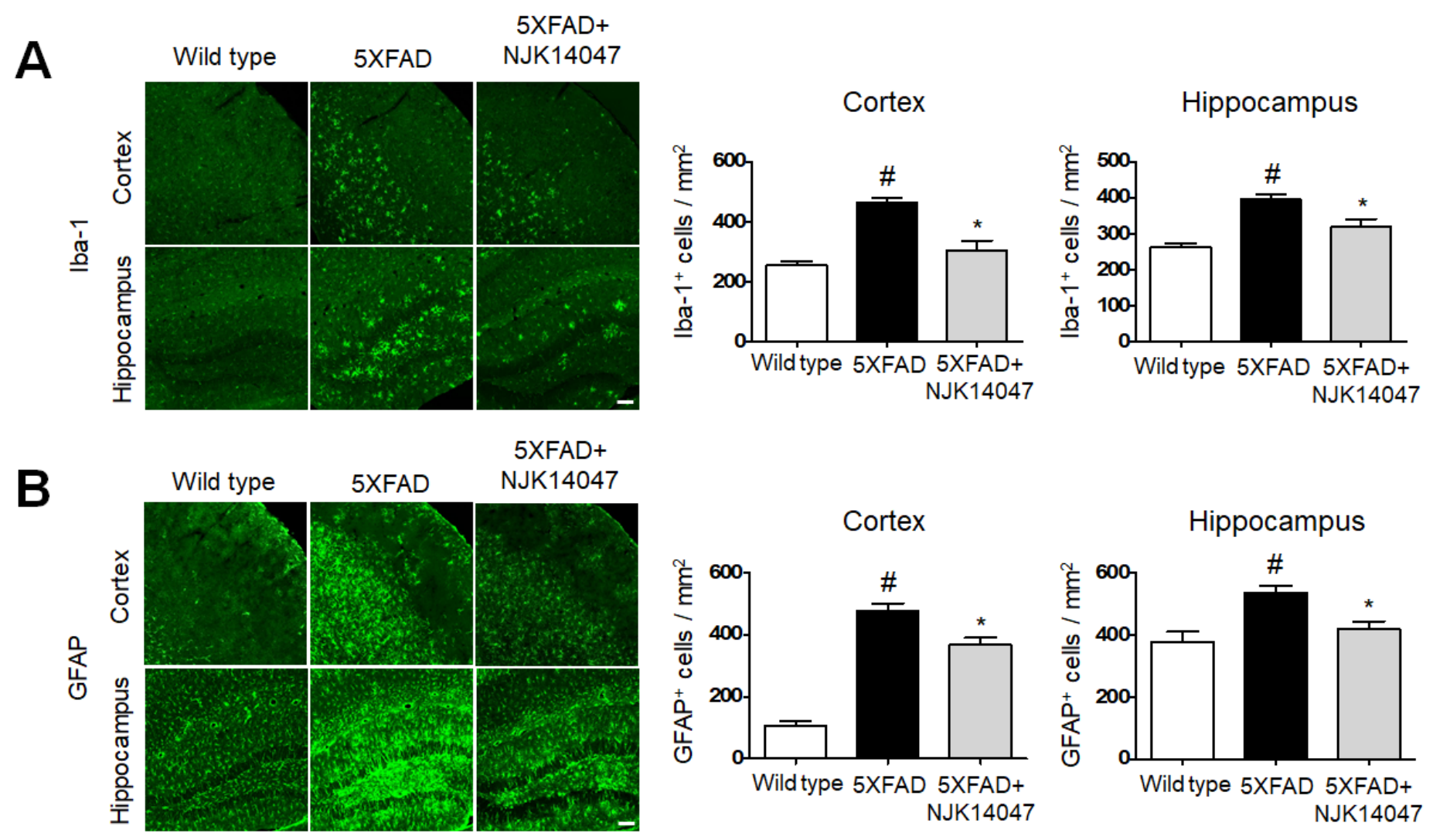

C

Cortex

Hippocampus

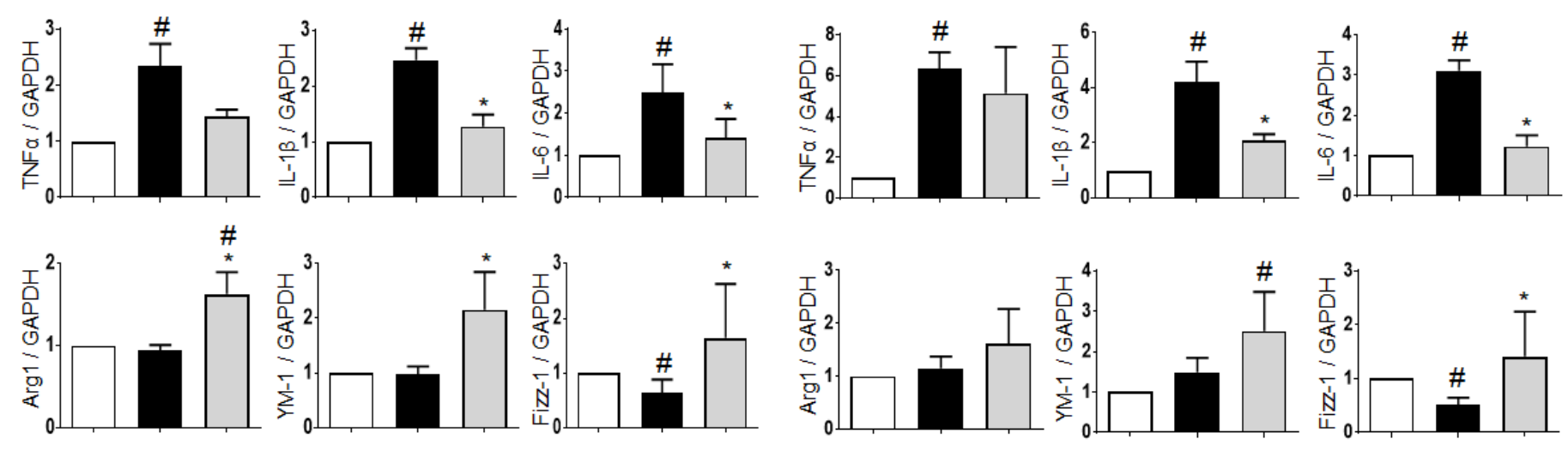

Wild type

5XFAD

5XFAD+NJK14047 


\section{Figure 4}

NJK14047 treatment from 6 months to 9 months of age downregulates neuroinflammation in the cortex and hippocampus. ( $A$ and $B$ ) Representative confocal microscope images of mouse brain sections stained with (A) Iba-1 antibody for labeling microglia and (B) GFAP antibody for labeling astrocytes. Iba1- and GFAP-positive cell counts per square millimeter were measured. Data are shown as mean \pm SEM (scale bar $=100 \mu \mathrm{m}, \mathrm{n}=5$ per group, one-way ANOVA). (C) Relative mRNA expression levels of M1 and M2 microglial markers were measured using qRT-PCR. Total mRNA was extracted in the mouse cortex and hippocampus. The mRNA expression levels were normalized to GAPDH and represented as the fold change with respect to the mean of the control group with SD ( $n=5-6$ per group, Kruskal-Wallis test). (\#P $<0.05$ vs. wild type; ${ }^{P}<0.05$ vs. $5 X F A D$ ) 


\section{Hippocampus}
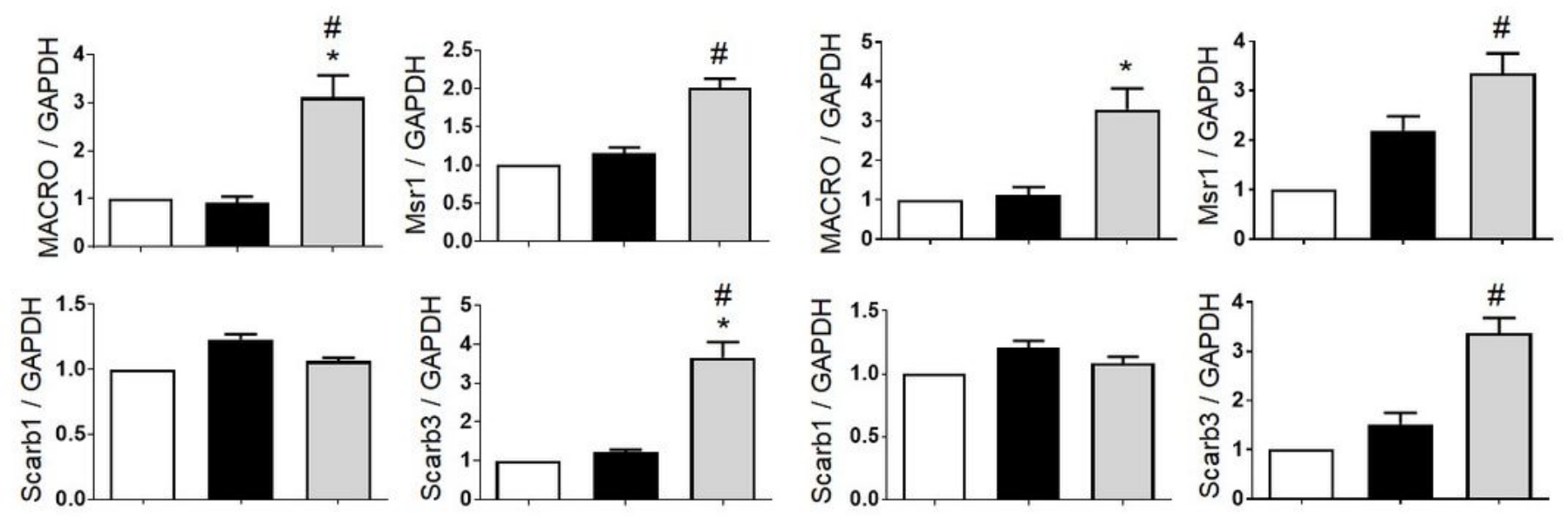

Wild type

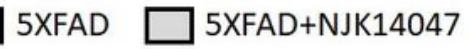

B

Thioflavin S

Iba-1

Merge
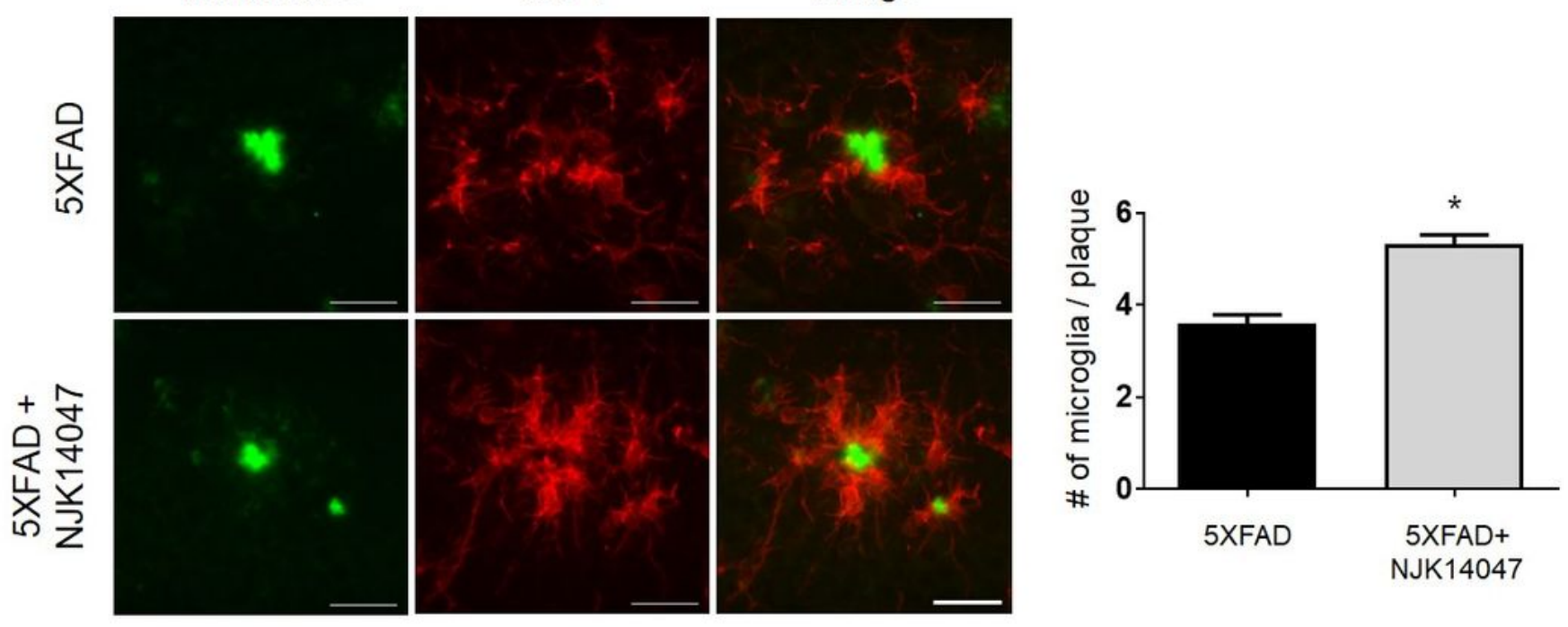

\section{Figure 5}

NJK14047 treatment from 6 months to 9 months of age up-regulates the expression levels of microglial phagocytic receptors and the $A \beta$ clearance function of microglia. (A) Relative mRNA expression levels of microglial phagocytic receptors in the cortex and hippocampus were measured using qRT-PCR. The expression levels were normalized to GAPDH levels and shown as the fold change with respect to the mean of the control group with SD ( $n=5-6$ per group, Kruskal-Wallis test). (B) Representative confocal 
microscope images for phagocytic microglia stained with thioflavin S and Iba- 1 antibody (scale bar $=30$ $\mu \mathrm{m})$. Microglial cell counts within the $20 \mu \mathrm{m}$ range of $A \beta$ plaque were quantified. Data were shown as mean \pm SEM ( $n=5$ per group, Student's t-test). (\#P<0.05 vs. wild type; $* P<0.05$ vs. $5 X F A D)$

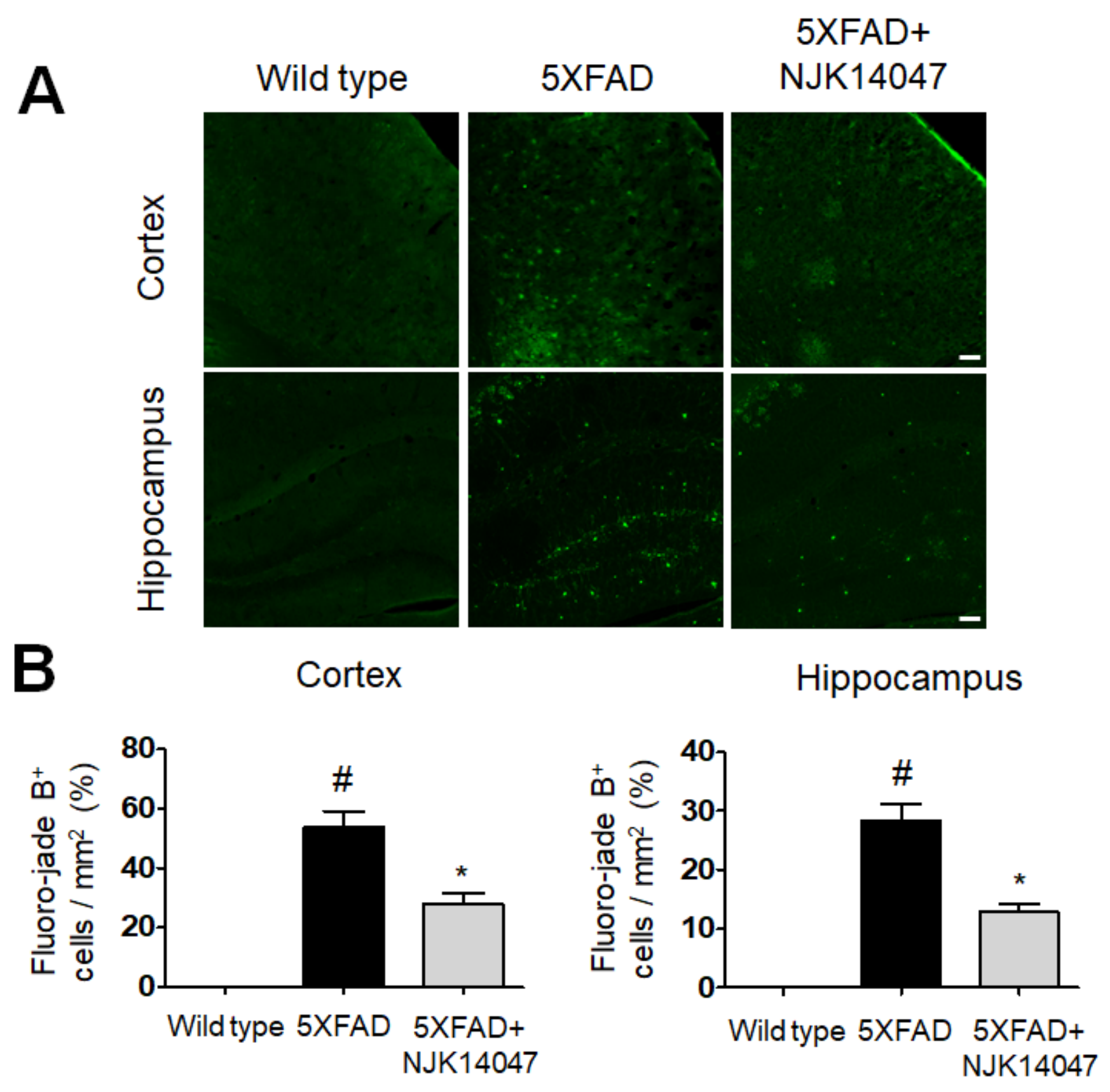

Figure 6

NJK14047 treatment from 6 months to 9 months of age inhibits neuronal deaths in the cortex and hippocampus. (A) Representative confocal microscope images of mouse brain sections stained with Fluoro-jade B dye for labeling degenerating neurons in the cortex and hippocampus (scale bar $=100 \mu \mathrm{m}$ ). 
(B) Quantitative analysis was performed with four sections in each mouse and four areas in each section. Fluoro-jade B-positive cells per square millimeter were quantitated. Data are shown as mean \pm SEM ( $n=5$ per group, one-way ANOVA). (\#P $<0.05$ vs. wild type; ${ }^{*} P<0.05$ vs. 5 XFAD)

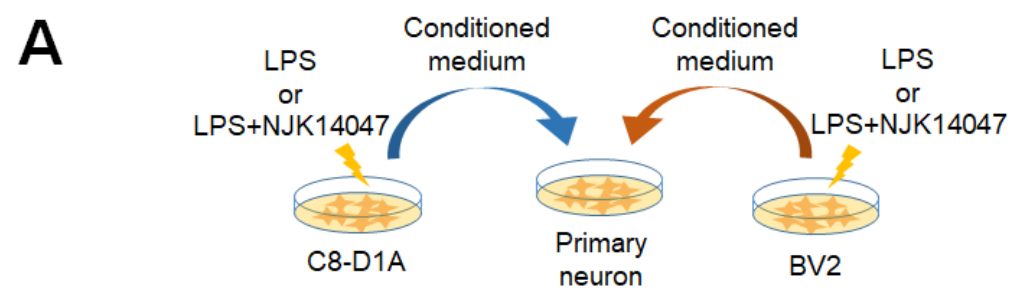

B
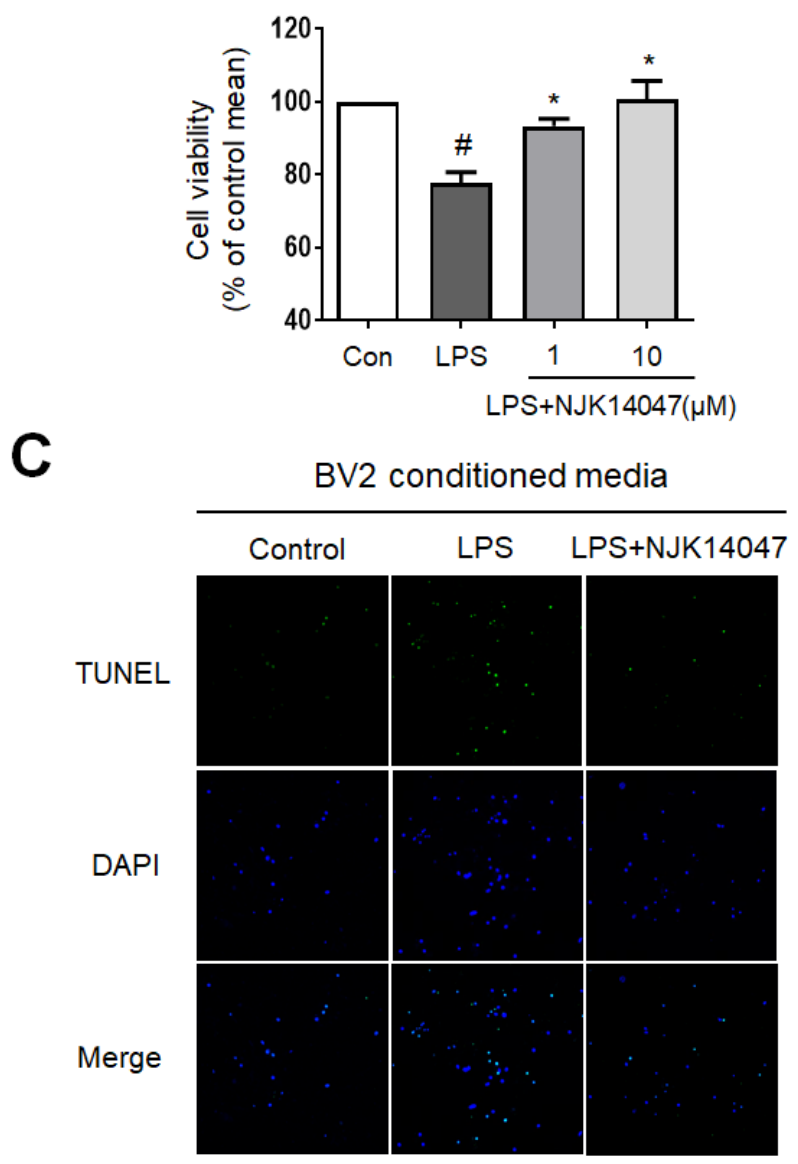

BV2 conditioned media

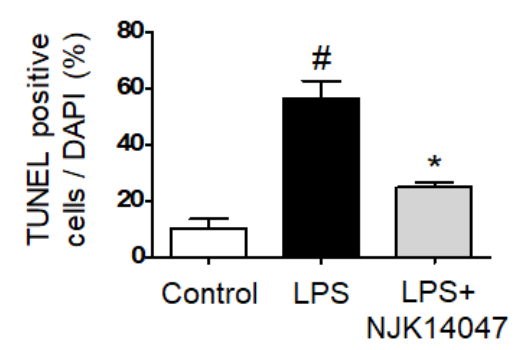

C8-D1A CM to neuron

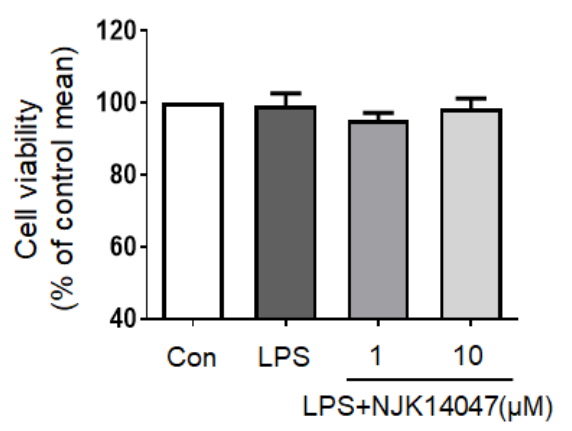

C8-D1A conditioned media

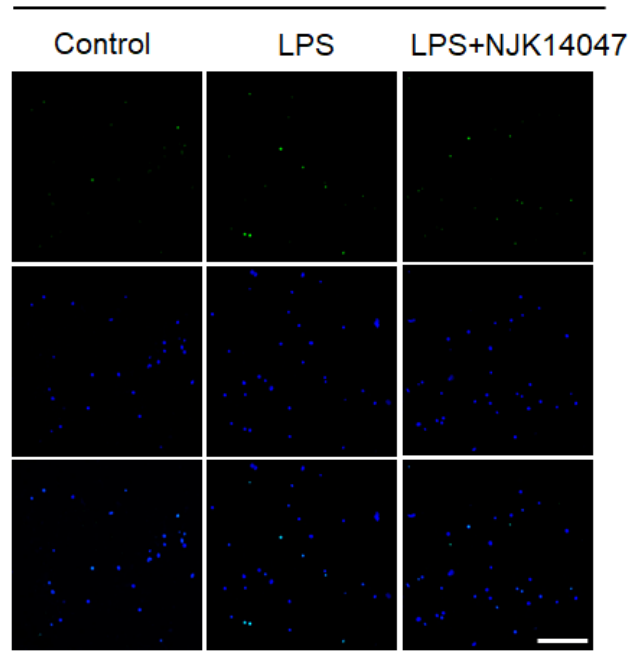

C8-D1A conditioned media

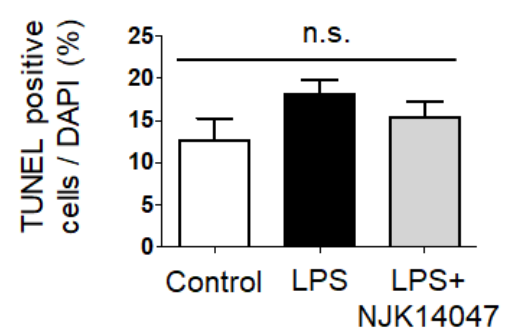

Figure 7

NJK14047 attenuates neurotoxicity of LPS-stimulated microglial cell line rather than that of LPSstimulated astrocyte cell line. (A) Scheme of the experiment. Microglial cell line BV2 and astrocyte cell 
line C8-D1A were seeded on 6-well plate at $5 \times 105$ cells/well. BV2 and C8-D1A were stimulated with 500 $\mathrm{ng} / \mathrm{ml}$ LPS alone or after $2 \mathrm{~h}$ of pre-treatment with either 1 or $10 \mu \mathrm{M}$ NJK14047. After $22 \mathrm{~h}$ of LPS stimulation, all media were changed to fresh neurobasal medium where cells were incubated for $24 \mathrm{~h}$. The conditioned medium was collected to treat primary neurons seeded on 48-well plate for cytotoxicity assay (B) or 24-well cover glass for TUNEL assay (C). (B) Viability of mouse primary neurons stimulated with BV2 and C8-D1A conditioned media. O.D. at $570 \mathrm{~nm}$ were normalized to the control group and shown as percentage of control mean $\pm S D$ ( $n=3$, Kruskal-Wallis test) (C) TUNEL assay for mouse primary neurons stimulated with BV2 and C8-D1A conditioned media. Apoptotic cells were quantified using four images in each cover glass. Data are shown as mean \pm SEM (scale bar $=100 \mu \mathrm{m}, \mathrm{n}=5$, one-way ANOVA). (\#P $<0.05$ vs. control group; ${ }^{*} P<0.05$ vs. LPS group) 


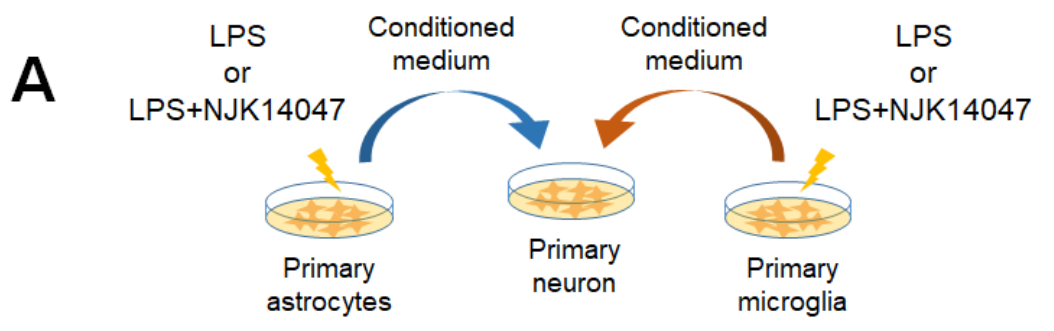

B

MCM to neuron

ACM to neuron

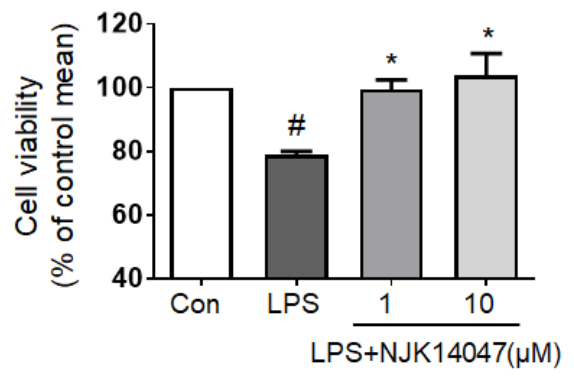

C

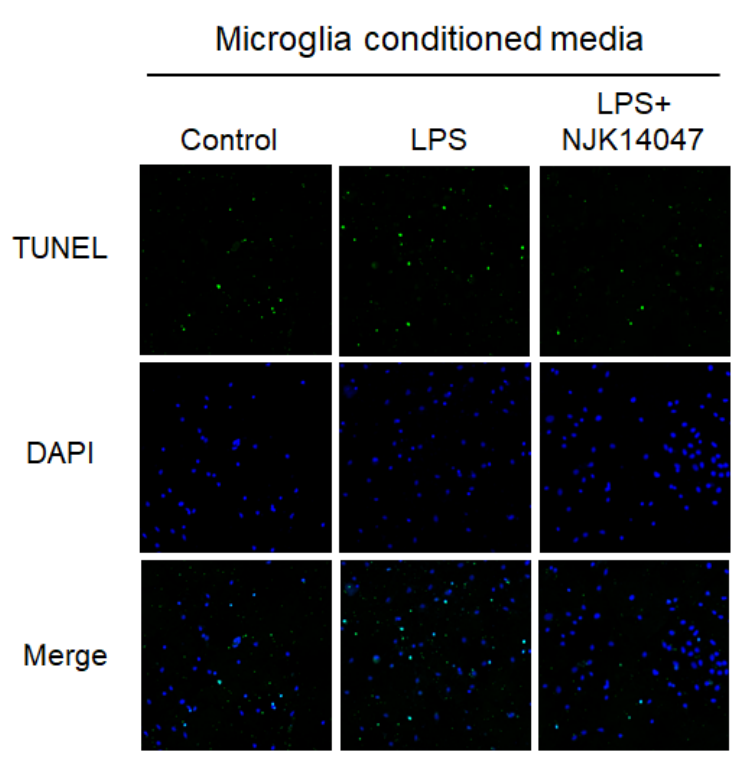

Microglia conditioned media
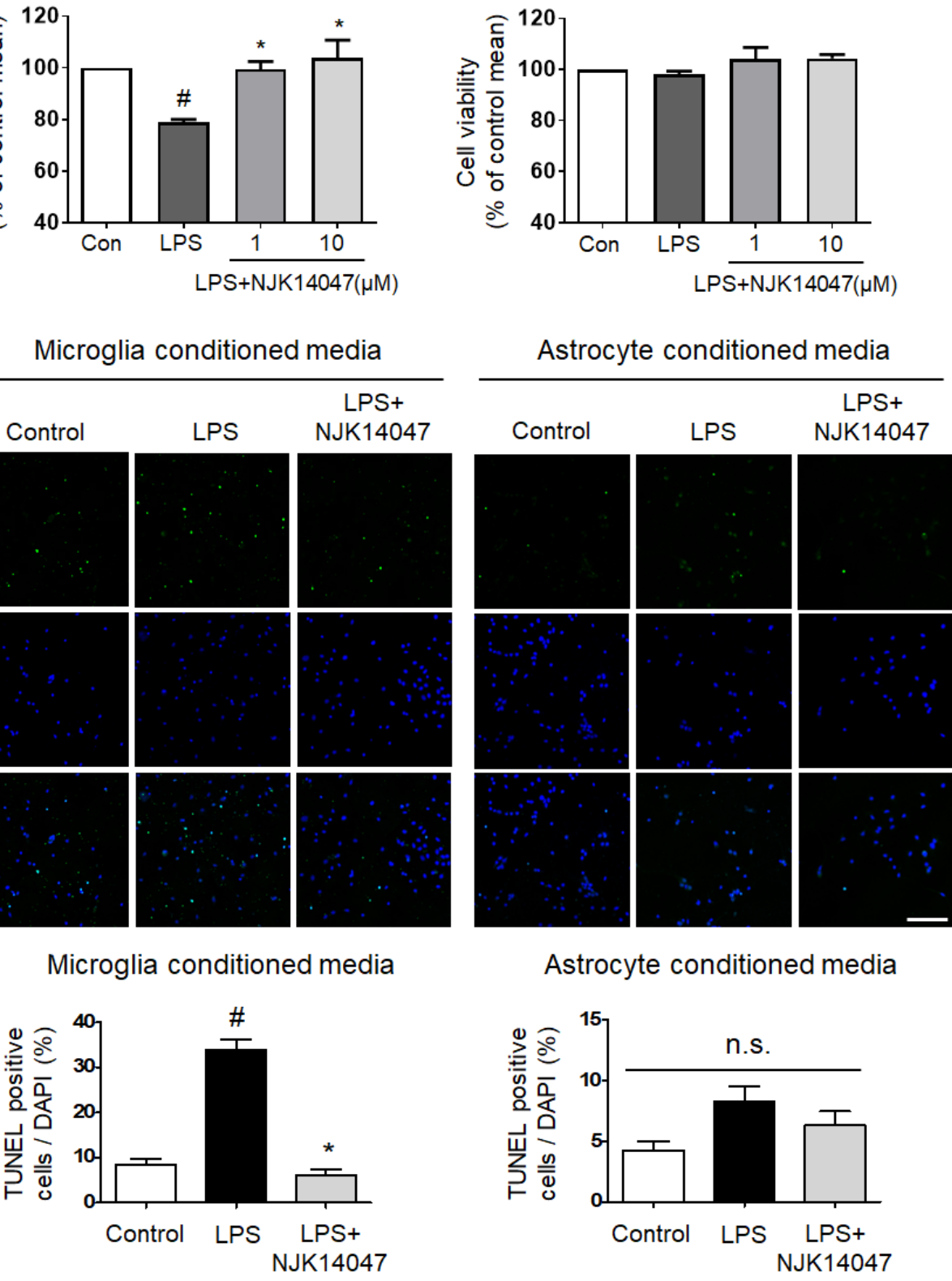

Astrocyte conditioned media

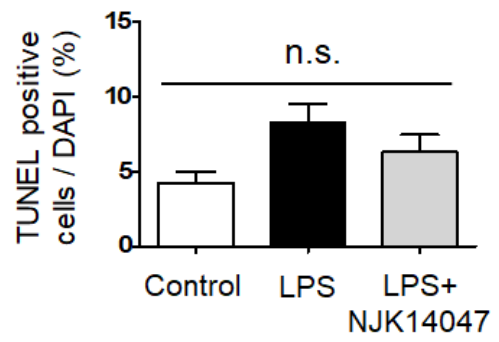

\section{Figure 8}

NJK14047 reduces LPS-stimulated microglial neurotoxicity rather than LPS-stimulated astrocyte neurotoxicity. (A) Scheme of the experiment. Mouse primary microglia and astrocytes were seeded on 6well plate at $5 \times 105$ cells/well and stimulated with $50 \mathrm{ng} / \mathrm{ml}$ LPS alone or after $2 \mathrm{~h}$ of pre-treatment with $10 \mu \mathrm{M}$ NJK14047. After $22 \mathrm{~h}$ of LPS stimulation, all media were changed to fresh neurobasal medium where cells were incubated for $24 \mathrm{~h}$. The conditioned medium was collected to treat primary neurons 
seeded on 24-well cover glass for TUNEL assay. (B) Viability of mouse primary neurons incubated with MCM and ACM for 24 h. O.D. at $570 \mathrm{~nm}$ were normalized to the control group and shown as percentage of control mean $\pm S D(n=3$, Kruskal-Wallis test) (C) TUNEL assay for mouse primary neurons stimulated with mouse primary microglia and astrocyte conditioned media. Apoptotic cells were quantified using four images in each cover glass. Data are shown as mean \pm SEM (scale bar $=100 \mu \mathrm{m}, n=5$, one-way ANOVA). (\#P< 0.05 vs. control group; $* P<0.05$ vs. LPS group) 